ARTICLE

https://doi.org/10.1038/s41467-019-12988-8

\title{
Foxp1 controls brown/beige adipocyte differentiation and thermogenesis through regulating $\beta 3-A R$ desensitization
}

Pei Liu'7, Sixia Huang ${ }^{2,7}$, Shifeng Ling ${ }^{2}$, Shuqin Xu², Fuhua Wang ${ }^{2}$, Wei Zhang ${ }^{2}$, Rujiang Zhou ${ }^{2}$, Lin $\mathrm{He}^{2}$, Xuechun $\mathrm{Xia}^{2}$, Zhengju Yao ${ }^{2}$, Ying Fan ${ }^{1}$, Niansong Wang ${ }^{1}$, Congxia Hu${ }^{3}$, Xiaodong Zhao ${ }^{4}$, Haley O. Tucker (1) ${ }^{5}$, Jiqiu Wang ${ }^{6 \star} \&$ Xizhi Guo ${ }^{1,2 \star}$

$\beta$-Adrenergic receptor ( $\beta$-AR) signaling is a pathway controlling adaptive thermogenesis in brown or beige adipocytes. Here we investigate the biological roles of the transcription factor Foxp1 in brown/beige adipocyte differentiation and thermogenesis. Adipose-specific deletion of Foxp1 leads to an increase of brown adipose activity and browning program of white adipose tissues. The Foxp1-deficient mice show an augmented energy expenditure and are protected from diet-induced obesity and insulin resistance. Consistently, overexpression of Foxp1 in adipocytes impairs adaptive thermogenesis and promotes diet-induced obesity. A robust change in abundance of the $\beta 3$-adrenergic receptor ( $\beta 3-A R$ ) is observed in brown/ beige adipocytes from both lines of mice. Molecularly, Foxp1 directly represses $\beta 3-A R$ transcription and regulates its desensitization behavior. Taken together, our findings reveal Foxp1 as a master transcriptional repressor of brown/beige adipocyte differentiation and thermogenesis, and provide an important clue for its targeting and treatment of obesity.

\footnotetext{
${ }^{1}$ Department of Nephrology, Shanghai Jiao Tong University Affiliated Sixth People's Hospital, Shanghai 200233, China. ${ }^{2}$ Bio-X Institutes, Key Laboratory for the Genetics of Developmental and Neuropsychiatric Disorders, Ministry of Education, Shanghai Jiao Tong University, Shanghai 200240, China. ${ }^{3}$ School of Biomedical Engineering, Shanghai Jiao Tong University, Shanghai 200240, China. ${ }^{4}$ Shanghai Center for Systems Biomedicine, Shanghai Jiao Tong University, Shanghai 200240, China. ${ }^{5}$ Institute for Cellular and Molecular Biology, University of Texas at Austin, Austin, TX 78712, USA. ${ }^{6}$ Department of Endocrinology and Metabolism, Ruijin Hospital, Shanghai Jiao Tong University School of Medicine, Shanghai 200025, China. ${ }^{7}$ These authors contributed equally: Pei Liu, Sixia Huang. *email: wangjq@shsmu.edu.cn; xzguo2005@sjtu.edu.cn
} 
E nergy homeostasis in mammals mainly is controlled by energy intake and expenditure. There are three types of adipose tissues: white adipose tissues (WAT), brown adipose tissues (BAT), and brown-like (beige) adipose tissue. Each are composed of morphologically and anatomically distinct adipocyte populations ${ }^{1,2}$. WAT acts to store energy in the form of triglycerides, whereas BAT and beige adipose tissues dissipate heat against cold or excessive $\operatorname{diet}^{3}$. Brown adipocytes uniquely express uncoupling protein-1 (UCP1), which is the functional marker of brown adipocytes and fuels oxidation and heat generation in the inner membrane of mitochondria. Brown adipocytes originate from $\mathrm{Myf5}^{+}$mesodermal progenitor cells, common progenitors of skeletal muscle ${ }^{4}$. Beige and brown adipocytes share similar morphological features as well as expression of UCP1. Both beige and brown adipocytes can be induced within WAT depots following cold exposure or $\beta$-adrenergic stimulation $^{5-8}$. These processes are termed browning of white adipocytes. In the context of the global obesity epidemic, mechanisms that activate brown or beige adipocytes may have significant clinical implications for metabolic disorders 9 .

Activated brown or beige adipocytes are induced to dissipate heat as adaptive thermogenesis under sympathetic nervous system (SNS) control ${ }^{10}$. Upon cold exposure, SNS releases adrenergic signals, such as noradrenaline or catecholamines, which activate the downstream pathway through binding adrenergic receptors in adipose tissues. A variable portfolio of $\alpha / \beta$ adrenergic receptors is then assembled within various mammalian tissues. Compared to the $\beta 1 / 2$ adrenergic receptors $(\beta 1 / 2-\mathrm{AR})$, the $\beta 3$ adrenergic receptor ( $\beta 3-\mathrm{AR})$ is relatively enriched in adipose tissues required for cold or diet-induced thermogenesis ${ }^{11,12}$. Adrenergic signaling triggers energy expenditure chronically via BAT thermogenesis ${ }^{13,14}$, and acutely via WAT fueling ${ }^{15,16}$. $\beta 3$ $\mathrm{AR}$ is primarily responsible for beige adipocyte induction in preexisting white adipocytes under adrenergic agonists stimulation, whereas $\beta 1$-AR mediates beige adipocyte generation after cold exposure $^{17}$. The role of $\beta 3$-AR agonists in beige induction and activation has also been demonstrated in human adipose tissues $^{18}$. Consequently, $\beta 3$-AR agonists can activate both typical brown and beige adipocytes through SNS, and thus, provide promising opportunities for anti-obesity treatments ${ }^{19}$.

Both the abundance and activity of $\beta 3-\mathrm{AR}$ in adipocytes are tightly regulated. For instance, the subjects carrying a Trp64Arg mutation within the $\beta 3-A R$ gene may be more susceptible to obesity $^{20-22}$. Consistent with this finding, mice devoid of the $\beta 3$ $A R$ gene are prone to deposit more fat than control mice ${ }^{23}$. In $o b /$ $o b$ mice, $\beta 3-A R$ expression is dramatically impaired in adipocytes $^{24}$. In fact, $\beta 3-A R$ has a unique expression dynamic in adipocytes termed desensitization. That is, $\beta 3-A R$ displays a shortterm decline in mRNA abundance upon exposure to $\beta 3-\mathrm{AR}$ agonists $^{25-28}$. This is distinct from the typical $\beta 2-A R$ desensitization pathway observed in cardiomyocytes ${ }^{29}$, which is cycled with $\beta 2$-AR protein between cell membrane and endosome through $\beta$-Arrestin protein. The biological significance of $\beta 3$-AR desensitization still is not fully recognized, and it remains unclear how $\beta 3-A R$ is transcriptionally regulated. Yet, these questions are absolutely critical in evaluating the role of $\beta 3$-AR in obesity treatment.

Brown/beige adipocyte differentiation and activation is controlled by sequential actions of transcription factors, including Ebf2, Prdm16, C/ebp $\beta$, PGC-1 $\alpha$ and PPAR $\gamma^{30-33}$. The Prdm16-C/ebp $\beta$ complex functions as a switch to determine the thermogenic program of brown/beige adipocytes ${ }^{34,35}$. On the other hand, Twist1 and Rip140 act to arrest BAT thermogenesis by repressing PGC-1 $\alpha$ activity $^{36,37}$. Foxhead P1 (Foxp1) typically acts as a transcriptional repressor in a variety of developmental pathways, including cardiomyocyte proliferation ${ }^{38,39}$, lung development ${ }^{40,41}$, lymphocyte differentiation ${ }^{42,43}$, glucose homeostasis ${ }^{44}$, endochondral ossification $^{45}$, and neuronal morphogenesis ${ }^{46-48}$. A recent study from our group further reveals an important role for Foxp1 in mesenchymal stem cell senescence ${ }^{49}$. In this study, we identify Foxp1 as a crucial component of the thermogenic program, which arrests brown/beige differentiation and thermogenesis through regulation of $\beta 3-\mathrm{AR}$ transcription in adipocytes.

\section{Results}

Foxp1 expression is sensitive to adrenergic stimuli. To examine the expression pattern of Foxp1 in adipose tissues, two representative subpopulations of adipocytes, interscapular BAT and subcutaneous WAT were investigated by immunofluorescence analyses. Foxp1 expression was strongly detected within brown and white adipocytes from 4 -week-old mice (Fig. 1a). Of the four isoforms $(\mathrm{A}-\mathrm{D})$ that are typically observed in a variety of mouse tissues $^{50}$, we detected primarily isoforms B and D in BAT, and isoforms A and B in WAT via western blotting analyses (Fig. 1b). In pheochromocytoma (PHEO) patients, beige adipocytes were induced inside omental WAT as a result of adrenergic stress under extremely excessive catecholamine expression ${ }^{51,52}$. In clinical samples from PHEO patients, we detected enrichment of FOXP1 expression in beige adipocytes in the vicinity of the vasculature within omental WAT (Fig. 1c and Supplementary Fig. 1a).

Then stromal vascular fraction (SVF) cells isolated from BAT of wild type mice were induced to brown adipocyte differentiation in vitro. After one day of induction, the elevated Foxp1 expression began to decline, whereas the expression of brown adipocyterelated genes (Ucp1,C/ebp $\alpha$ and $P P A R \gamma$ ) was progressively upregulated (Fig. 1d). Similarly, qPCR analyses confirmed the downregulation of Foxp1 during the 8-hour course of white adipogenic induction in 3T3-L1 cells (Supplementary Fig. 1b), which was consistent with previous findings ${ }^{53}$. These observations indicate that Foxp1 is extensively expressed in adipocytes, and its expression shows a transit peak at the very earliest stage of adipocyte differentiation.

Next, we examined the dynamics of Foxp1 expression following stimulation of adrenergic signaling. When mice were challenged by cold exposure $\left(4^{\circ} \mathrm{C}\right)$ overnight, Foxp 1 expression in BAT was upregulated (Fig. 1e, f). At the cellular level, when brown adipocytes derived from SVF of mice or humans were exposed to CL-316,243 $(0.1 \mu \mathrm{M})$ for up to $8 \mathrm{~h}$, Foxp1 expression progressively inclined, whereas $\beta 3-A R$ expression inversely declined (Fig. 1g, h). Consistently, the expression of $\beta 3-A R$ behaved as a typical desensitization process at the transcriptional level in responsive to adrenergic signaling in vivo and in vitro (Fig. 1e, g, h). These observations suggest that Foxp1 expression in adipose adipocytes is dynamic, and can be induced by adrenergic signaling with an inverse expression pattern of $\beta 3-A R$.

Previous studies have demonstrated that MAPK kinases, including Erk1/2 and p38, could mediate adrenergic signaling in adipocytes ${ }^{15,54}$. To test if either of these kinases mediates induction of Foxp1 by the $\beta 3$-AR agonist, we first evaluated the dynamics of Foxp1 expression in an 8-h course of adipogenic cultures of 3T3-L1 cells. Briefly, the cells were stimulated with CL-316,243 alone or in combination with SB202190 (p38 kinase inhibitor), FR180204 or SCH772984 (Erk1/2 inhibitors). As shown in Fig. 1i, the induction of Foxp1 transcripts by the $\beta 3-\mathrm{AR}$ agonist was markedly arrested by FR180204 and SCH772984, whereas it was exaggerated by SB202190. This suggested that Erk1/2 is necessary for Foxp 1 induction by adrenergic stimulation. These results were further validated by western blotting following exposure of brown adipocytes to CL-316,243 and SCH772984 (Fig. 1j). Together our findings indicate that Foxp1 
a

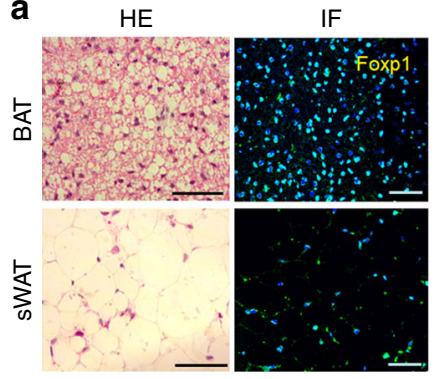

b

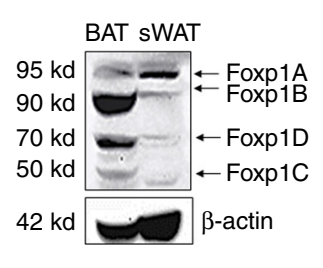

e

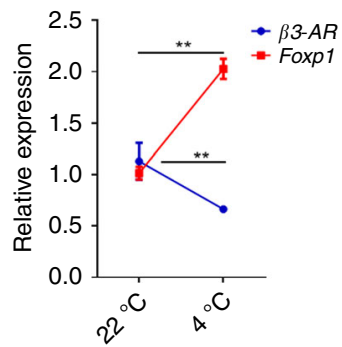

f

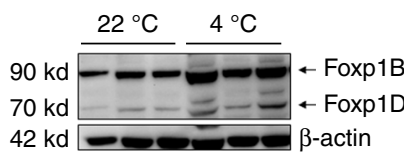

i

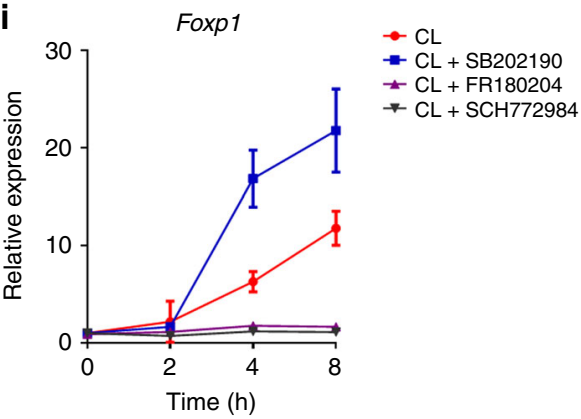

C

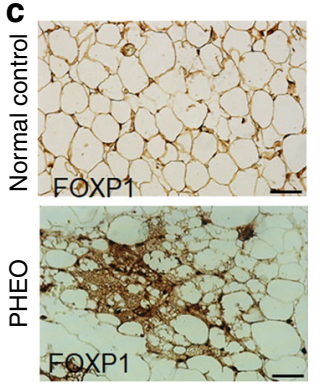

d

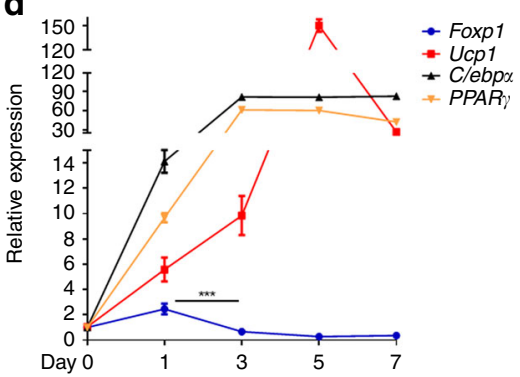

h Human BAT $+0.1 \mu \mathrm{M} \mathrm{CL}$

g Murine BAT $+0.1 \mu \mathrm{M} \mathrm{CL}$
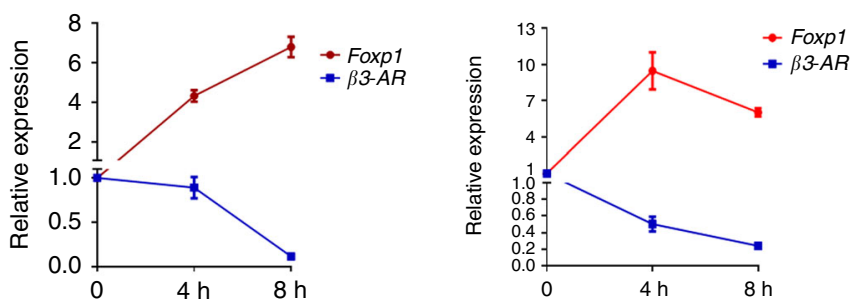

j

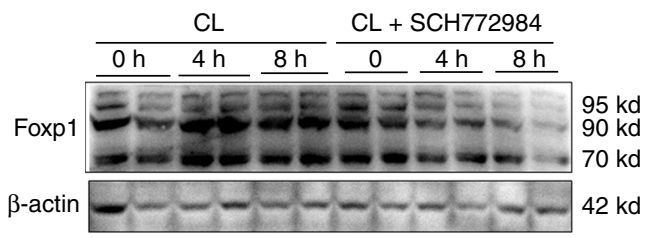

Fig. 1 Foxp1 expression in adipocytes is induced by adrenergic stimuli. a H\&E staining and immunofluorescence (IF) analysis for the Foxp1 expression in BAT and sWAT from wild type mice at age of 4 weeks. DAPI, blue staining for nucleus; green color for Foxp1 expression. Bar, $50 \mu$ m. b Western blotting showed the four isoforms (A, B, D, C) of Foxp1 protein in BAT and sWAT from wild type mice at age of 2 months. c IHC analysis of FOXP1 expression in biopsies from PHEO patients and normal controls. Bar, $10 \mu \mathrm{m}$. d qPCR analysis of expression of Foxp1 and brown adipocyte markers (C/ebp $\alpha$, Ppar $\gamma$, and Ucp1) during the time course of brown adipocyte differentiation from SVFs. e qPCR analysis of Foxp1 and $\beta 3-A R$ expression in BAT in mice with overnight $4{ }^{\circ} \mathrm{C}$ cold exposure. $n=3$ biologically independent samples. $\mathbf{f}$ Western blotting of Foxp1 in BAT from mice above (e). $\mathbf{g}$, $\mathbf{h}$ qPCR analysis of Foxp 1 and $\beta 3-A R$ expression in brown adipocytes differentiated from murine ( $\mathbf{g}$ ) and human SVF (h) during an 8-hour CL-316,243 (0.1 $\mu \mathrm{M})$ treatment as indicated. $n=3$ biologically independent experiments. i Foxp1 expression profile in adipocytes derived from 3T3-L1 cells during an 8-h time course, stimulated by CL$316,243(0.5 \mu \mathrm{M})$ with or without SB202190 (p38 kinase inhibitor, $10 \mu \mathrm{M}$ ), FR180204 (Erk1/2 inhibitors, $1 \mu \mathrm{M}$ ) and SCH772984 (Erk1/2 inhibitors, $10 \mu \mathrm{M}$ ), respectively. $n=3$ biologically independent experiments. $\mathbf{j}$ Western blotting for Foxp1 in brown adipocytes derived from SVF, which were stimulated by CL$316,243(0.1 \mu \mathrm{M})$ with or without SCH772984 $(10 \mu \mathrm{M})$ for $8 \mathrm{~h} .{ }^{\star} P<0.05 ;{ }^{\star \star} P<0.01$; ${ }^{\star \star \star} P<0.001$; error bar, mean $\pm \mathrm{SEM}$

expression in adipocytes is induced by a $\beta 3-\mathrm{AR} /$ Erk $1 / 2$ signal cascades.

Foxp1 deletion potentiates brown adipocyte differentiation. As a next step in investigating the function of Foxp1 in brown adipocyte differentiation, we generated $F o x p 1_{M y f 5}{ }^{\Delta / \Delta}$ mice by crossing Foxp1 $1^{f l f l}$ with Myf5-Cre to facilitate deletion of Foxp1 specifically within BAT progenitors ${ }^{4}$ (Fig. $2 \mathrm{c}$ and Supplementary Fig. 1c). At the early postnatal stage, The BAT appeared normal in morphology and histology in Foxp $1_{M y f 5}{ }^{\Delta / \Delta}$ mice as compared to controls (Fig. 2a and upper panel in Fig. 2b). However, immunohistochemistry (IHC) analysis revealed a relative increase of UCP1 expression in the BAT of $F o x p 1_{M y f 5}{ }^{\Delta / \Delta}$ mice (Fig. 2b, lower panel), which was validated by western blotting (Supplementary Fig. 1c). In addition, qPCR detected elevated expression of BAT-related (Ucp1, Dio2, Prdm16, Tbx1, PPAR ) and mitochondrial (Cox7a1, Cox8b, Cox $5 b$, Cox2, Cpt2) genes in Foxp1${ }_{M y f 5}{ }^{\Delta / \Delta}$ BAT (Fig. 2d). These observations indicate that Foxp1 deletion accelerates brown adipocyte differentiation from progenitor cells.

Foxp1 deficiency promotes adipose tissue browning. To observe any potential influence of Foxp1 deficiency on beige adipocyte differentiation in vivo, we eliminated Foxp1 in adipocytes with Adiponectin-Cre $e^{55}$, which thereafter designated as $\mathrm{Foxp}_{A d} \Delta / \Delta$. Compared to control littermates, Foxp $1_{A d} \Delta / \Delta$ knockout mice appeared normal size (Fig. 2e), but was slightly smaller in BAT and sWAT depots (Fig. 2f). H\&E staining detected a reduction in the size of white adipocytes in $\operatorname{Foxp}_{A d} \Delta / \Delta$ sWAT in relative to controls (Fig. 2g). Typical browning characteristics of adipose tissues were detected by anti-UCP1 IHC of sWAT in Foxp $1_{A d} \Delta / \Delta$ mice exposed to $6-\mathrm{h} 4^{\circ} \mathrm{C}$ challenge (Fig. $2 \mathrm{~h}$ ). The activation of brown or beige adipocytes were observed by the elevated expression of a broad panel of thermogenic or BAT-selective marker genes (Ucp1, PGC-1 $\alpha, P G C-1 \beta$, Dio2, Cidea, Otop1, Prdm16, PPARa, Cox7a1, Eva1, Cox4il, Cox8b) (Fig. 2j, k), 

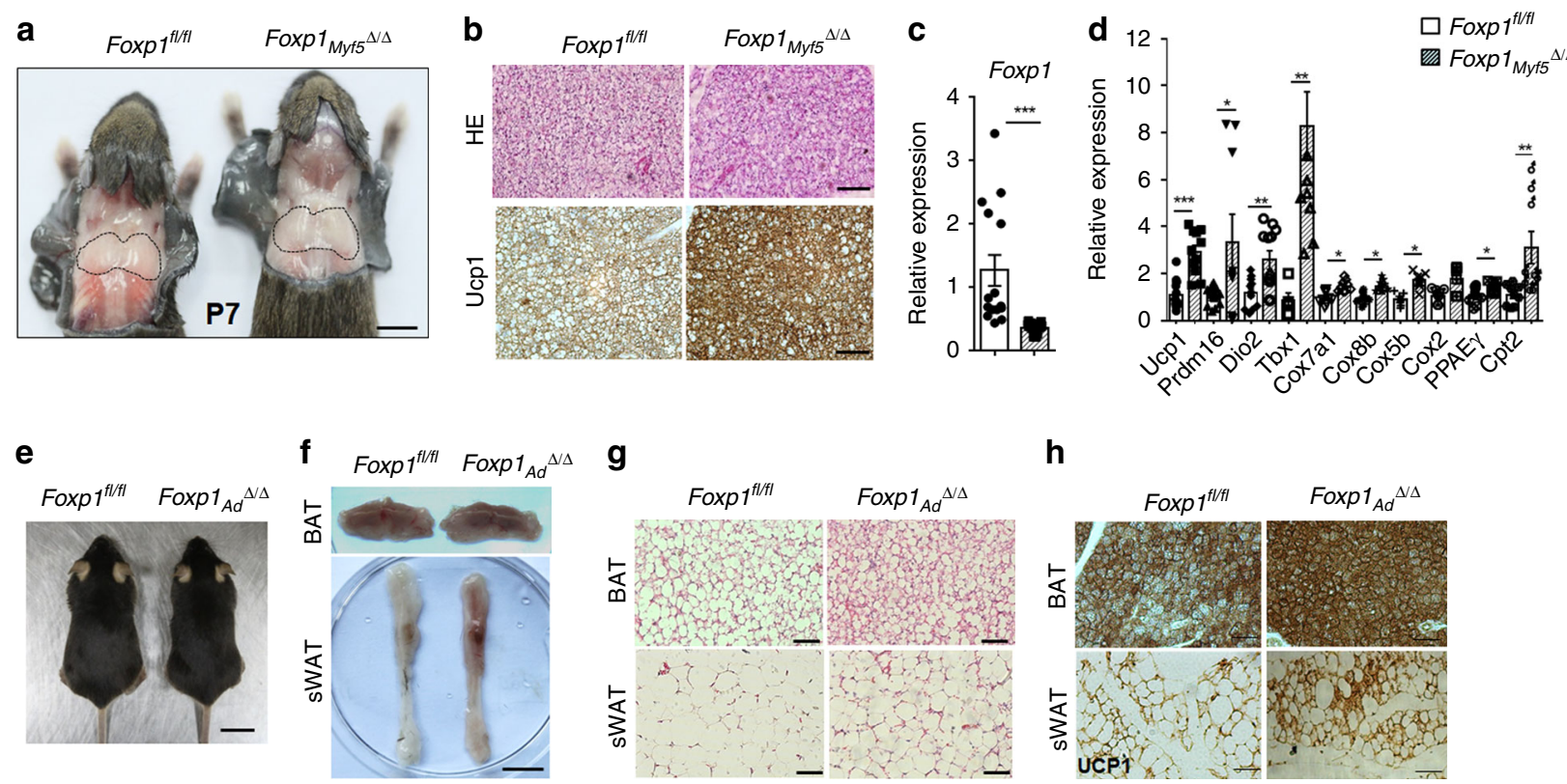

g

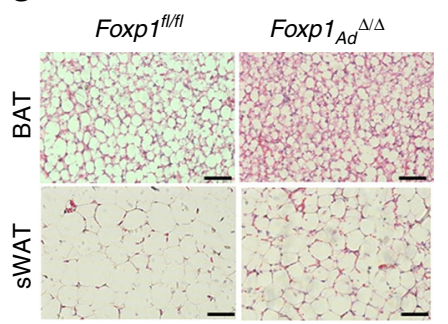

$\mathbf{h}$

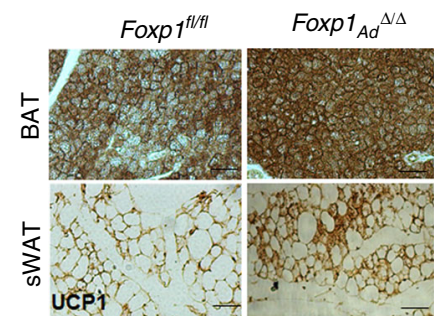

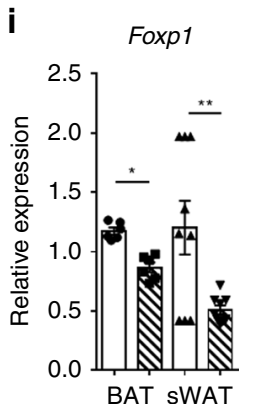
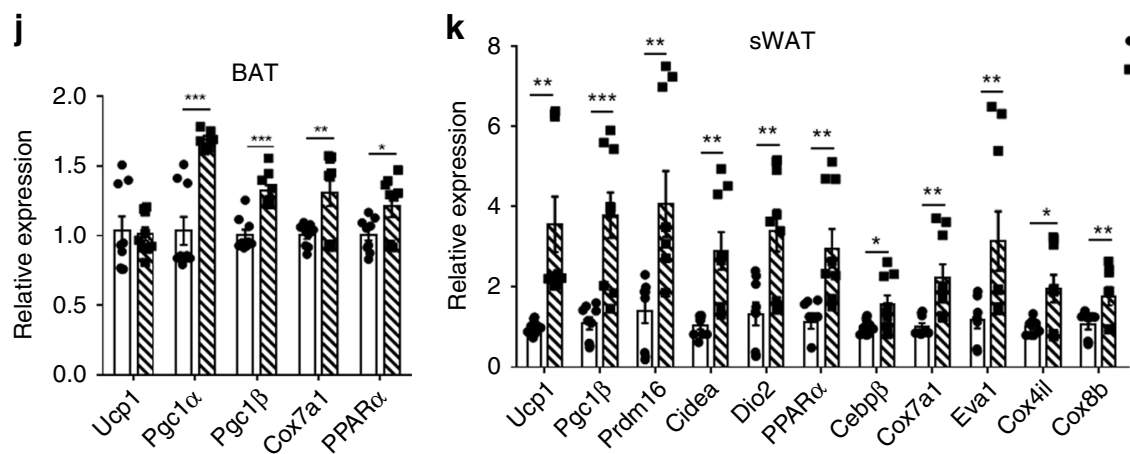

- Foxp $1^{f l / f l}$

- oxp $_{A d}{ }^{4 / 4}$

Fig. 2 Foxp1 deficiency promotes brown and beige adipocyte activation. a Representative dorsal view of BAT depot in Foxp ${ }^{f l / f l}$ and Foxp ${ }_{M y f 5}{ }^{\Delta / \Delta}$ mice at postnatal day 7. Bar, $1 \mathrm{~cm}$. b HE (upper panel) and IHC staining (lower panel) with UCP1 antibody for BAT in (a). Bar, $10 \mu \mathrm{m}$. c, d qPCR analysis of Foxp1 and BAT-selective markers for BAT depot. $n=5$ biologically independent samples. e Representative view of 3-month-old Foxp fl/fl and Foxpl ${ }_{A d} d / \Delta$ mice. Bar, $2 \mathrm{~cm}$. f Representative view of BAT (upper panel) and sWAT depot (lower panel) in (e). $\mathbf{g}$ HE staining for BAT and sWAT in mutant mice. Bar, $50 \mu \mathrm{m}$. $\mathbf{h}$ IHC analysis with UCP1 antibody for BAT and sWAT upon 6-h cold exposure. Bar, $50 \mu \mathrm{m}$. i-k qPCR for expression of Foxp1 and BAT-selective genes in BAT and sWAT from Foxp $f^{f / f l}$ and Foxp1 ${ }_{A d} \Delta / \Delta$ mutant mice. $n=4$ biologically independent mice/each group. ${ }^{\star} P<0.05$; ${ }^{\star \star} P<0.01$; ${ }^{\star \star \star} P<0.001$; error bar, mean \pm SEM

whereas the Foxp1 expression was significantly decreased in mutant BAT and sWAT (Fig. 2i).

We also ablated Foxp1 in adipocytes by aP2-Cre, which was also extensively utilized for adipose-specific knockout models ${ }^{56}$. Foxp1 was efficiently reduced at the mRNA and protein levels in BAT and sWAT from Foxp $1_{a P 2}{ }^{\Delta / \Delta}$ mice (Supplementary Fig. 1d, e). Similar to $\operatorname{Foxp}_{A d}{ }_{A d}{ }^{\Delta}$, Foxp $1_{a P 2}{ }^{\Delta / \Delta}$ mice were also smaller size in adipose depot (Supplementary Fig. 2a, b). H\&E staining and transmission electron microscopic (TEM) analysis revealed smaller lipid droplets as well as relative enrichment in mitochondria within brown and beige adipocytes of the mutant mice (Supplementary Fig. 2c, d), as evidenced by increased expression of mitochondrial specific gene, Co.1 (Supplementary Fig. 2e). In addition, adipose tissue browning in Foxp1 ${ }_{a P 2} \Delta / \Delta$ mice were observed by anti-UCP1 immunofluorescence (Supplementary Fig. 2f), elevated expression of BAT-selective genes (Supplementary Fig. 2g), as well as western blotting with antiUCP1 and anti- PGC-1a (Supplementary Fig. 2h). Foxp1deficient adipose tissues also displayed elevated expression of lipolytic genes, including $H s l, A t g l, A c s l$, and Acoxl (Supplementary Fig. 2i), and augmented p38 and HSL phosphorylation
(Supplementary Fig. 2j) - key mediators or targets of $\beta 3$-AR signaling for lipolysis ${ }^{13}$. Taken with our observations in Foxp $1_{M y f 5} \Delta / \Delta, F o x p 1_{A d}{ }^{\Delta / \Delta}$ and Foxp $1_{a P 2}{ }^{\Delta / \Delta}$ mice, these findings suggest that Foxp1 suppresses both brown and beige adipocyte differentiation and thermogenesis.

To test for a potential cell-autonomous effect of Foxp1 loss on brown/beige differentiation, stromal vascular fraction (SVF) cells were isolated from BAT or sWAT depots of $F o x p 1_{a P 2}{ }^{\Delta / \Delta}$ and control mice and then induced for brown adipocyte differentiation in vitro. We observed advanced brown/beige adipocyte differentiation from the Foxp1-deficient SVF progenitors (Supplementary Fig. 3a, e). Further, expression of a set of BATselective (Ucp1, PPAR $\alpha, P G C-1 \alpha, P G C-1 \beta$, Otop1, Dio2, Tbx1) and mitochondrial (Cox7a1, CytoC, Cpt2, Cox2, Cox5b, Cox8b) transcripts were significantly upregulated in cultured brown and beige adipocytes (Supplementary Fig. 3b-h). We next analyzed oxygen consumption rates (OCR) of SVF-derived adipocytes. Brown adipocytes from Foxp $1_{a P 2} \Delta / \Delta$ mutant mice exhibited higher total and uncoupled OCR (Supplementary Fig. 3i). Similarly, OCR was each significantly elevated in the white adipocytes from $\mathrm{Foxp}_{a P 2}{ }^{\Delta / \Delta}$ relative to controls (Supplementary 

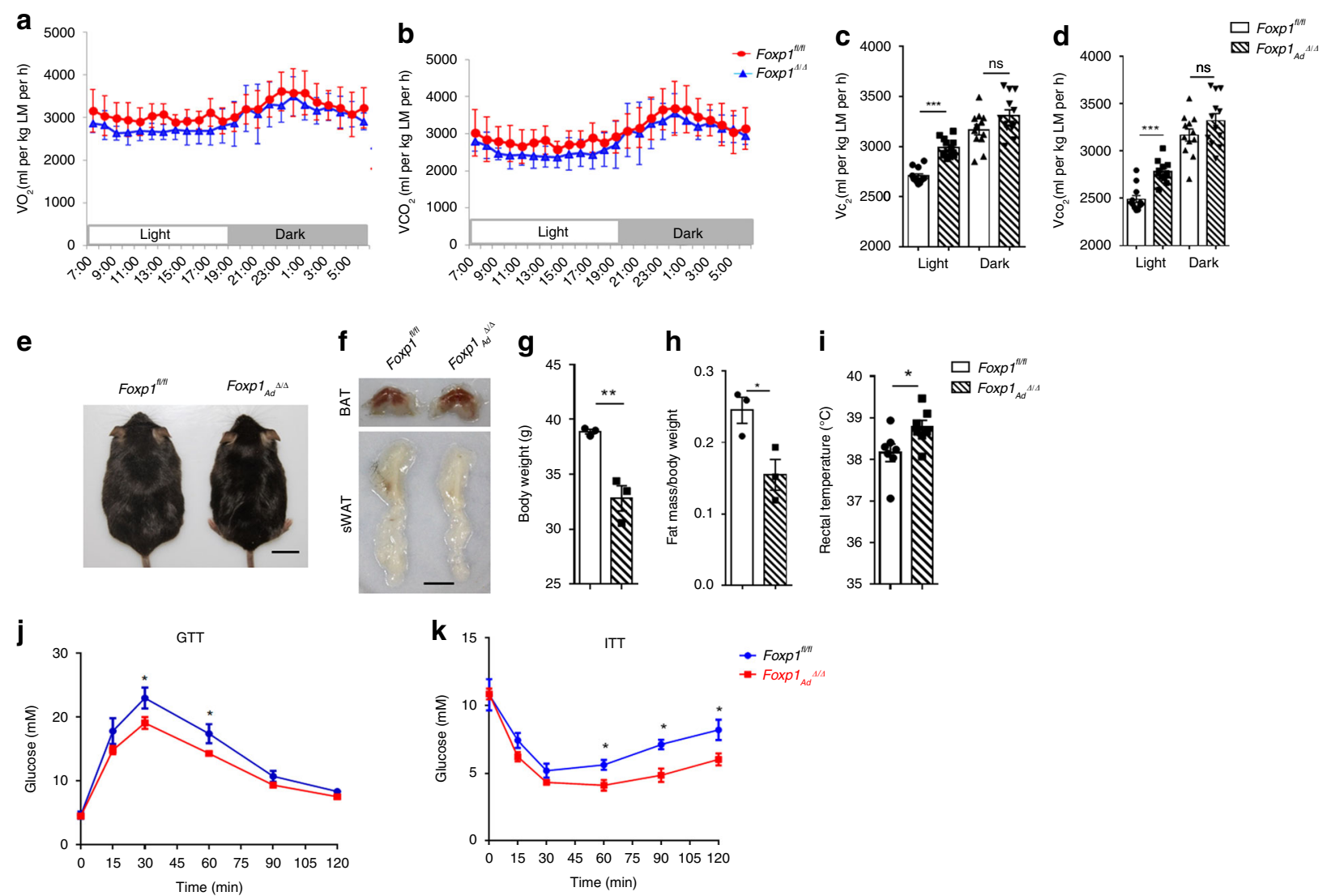

Fig. 3 Foxp 1 deletion elevates energy expenditure and resists to HFD-induced obesity. $\mathbf{a}, \mathbf{b} \vee \mathrm{VO}_{2}$ and $\mathrm{VCO}_{2}$ of Foxp $\mathrm{fl}^{\mathrm{f} / \mathrm{fl}}$ and $\mathrm{Foxp} \mathrm{Ad}_{\mathrm{Ad}} \mathrm{A} / \mathrm{\Delta}$ mice in metabolic cages at age of 3 months old. $n=7$ biologically independent mice/each group. c, d Quantification of $\mathrm{O}_{2}$ and $\mathrm{CO}_{2}$ consumption in light and dark. e Dorsal view of Foxp $1^{f / / f l}$ and Foxp1 ${ }_{A d} \Delta / \Delta$ mice after 8-week feeding with HFD at age of 6 months old. Bar, $2 \mathrm{~cm}$. $\mathbf{f}$ Representative depot of BAT and sWAT in HFDfed mice (e). Bar, $1 \mathrm{~cm}$. $\mathbf{g}$ Body weight of HFD-fed mice. h Relative adiposity of HFD-fed mice. i Statistics of rectal temperature of HFD-fed mice. $\mathbf{j}, \mathbf{k}$ GTT and ITT of HFD-fed mice. ${ }^{\star} P<0.05$; ${ }^{\star \star} P<0.01 ;{ }^{\star \star \star} P<0.001 ; n=7$ biologically independent mice/each group; error bar, mean \pm SEM

Fig. 3j). These lines of evidence indicate that Foxp1 controls brown/beige adipocyte differentiation and thermogenesis in a cell-autonomous manner.

Foxp1 deletion augments thermogenesis and energy expenditure. As BAT activation and sWAT browning are features of thermogenesis and energy expenditure, we next examined energy metabolism in mutant mice. Foxp $1_{A d} \Delta / \Delta$ and Foxp $1_{a P 2}{ }^{\Delta / \Delta}$ mice exhibited higher rates of oxygen consumption and carbon dioxide production, expended more energy (Fig. 3a-d and Supplementary Fig. 4a-d). And more, infrared imaging revealed that Foxp $1_{a P 2} \Delta / \Delta$ mice displayed higher skin temperatures as compared to Foxp 1 fl/fl controls after 1-hour exposure to $4^{\circ} \mathrm{C}$ (Supplementary Fig. 4e, f). These observations are consistent with that Foxp1 deficiency increases energy expenditure and thermogenesis in vivo.

To examine the long-term effect of Foxp1 loss on energy balance, mutant mice were subjected to a high-fat diet (HFD). Foxp $1_{A d}{ }^{\Delta / \Delta}$ mice appeared leaner in body, with smaller in adipose depots (Fig. 3e, f), and gained less body weight than littermates after 8-week HFD feeding starting at age of 6 months old (Fig. 3g). The reduction in weight primarily resulted from a decrease in adiposity (Fig. 3h). Meanwhile, $\operatorname{Foxp}_{A d}{ }^{\Delta / \Delta}$ mice appeared relatively high rectal temperature as compared to controls (Fig. 3i). Foxp $1_{A d} \Delta / \Delta$ mutant mice also retained better glucose tolerance and higher insulin sensitivity following HFD feeding, as evidenced by glucose tolerance test (GTT) and insulin tolerance test (ITT) scores (Fig. 3j, k). Foxp $1_{a P 2}{ }^{\Delta / \Delta}$ knockout mice displayed similar phenotypes with $F o x p 1_{A d} \Delta / \Delta$ mice, including resistance to HFD-induced obesity as well as improved glucose metabolism and insulin sensitivity after HFD feeding (Supplementary Fig. 4g-l). Our findings suggest that BAT activation and WAT browning in Foxp1-deficient mice results in marked improvement of glucose metabolism and protects mice from HFD-induced obesity (potentially as a result of elevated thermogenesis).

Overexpression of Foxp1 represses adaptive thermogenesis. To further examine the effect of Foxp 1 on energy expenditure, we generated aP2-Foxp1 transgenic mice in which Foxp1 cDNA overexpression was driven by a $5.4 \mathrm{~kb} \mathrm{Fabp4} \mathrm{(aP2)}$ gene promoter/enhancer cassette. Overexpression of Foxp1 was validated by qPCR and western blotting of four independent founders (Supplementary Fig. 5a, b). We then employed Thermo Mouse, an Ucp1-Luciferase (Ucp1-Luc) reporter mouse ${ }^{57}$, as a tool to detect adaptive thermogenesis in brown and beige adipose tissues under cold exposure or adrenergic stimulation. Two representative strains of aP2-Foxp1;Ucp1-Luc transgenic mice displayed lower luciferase activities in BAT and sWAT compared to Ucp1-Luc controls when challenged with $4^{\circ} \mathrm{C}$ cold exposure for $6 \mathrm{~h}$ (Fig. $4 \mathrm{a}$ ). Transgenic BAT depots appeared less brown in color and larger in cellular size as compared to wild type controls (Fig. 4b, c). We also observed that our transgenic mice displayed impaired thermogenesis under cold exposure, as evidenced by decreased UCP1 
a

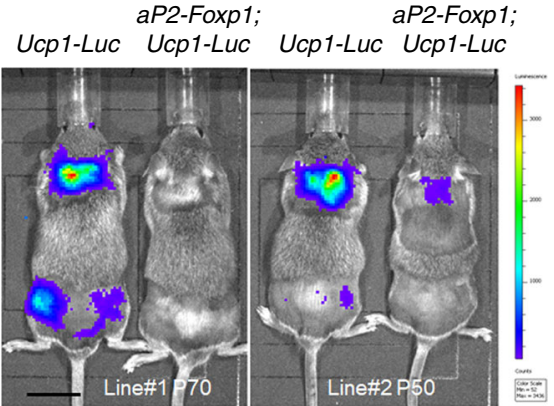

d

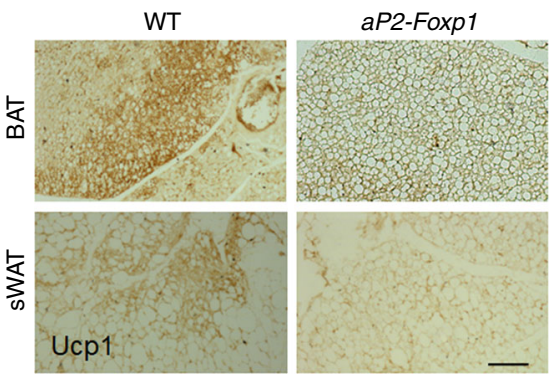

b

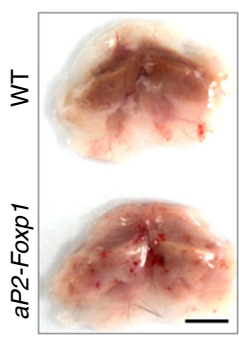

C

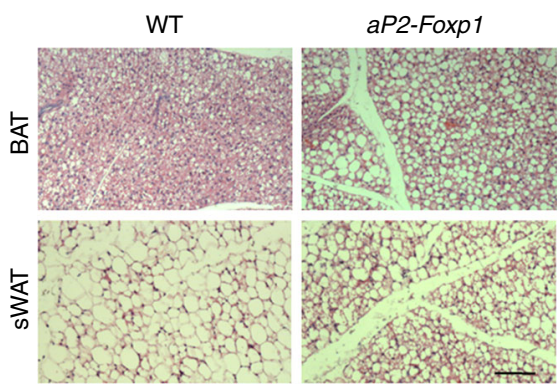

e

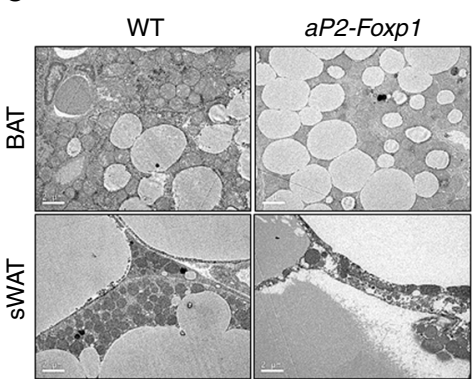

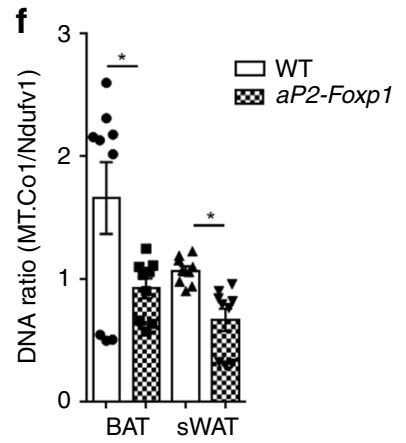

g

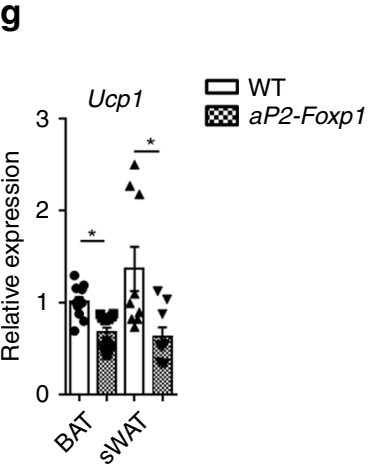

$\mathbf{k}$

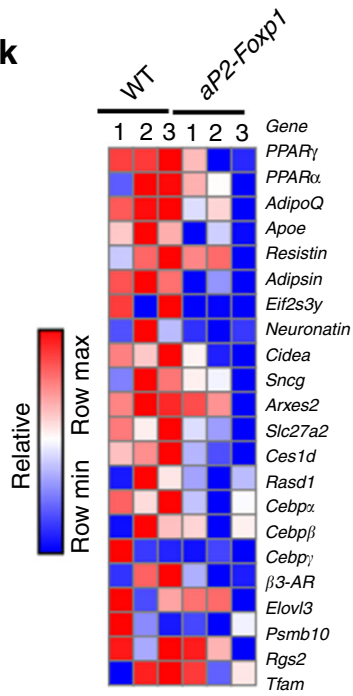

h

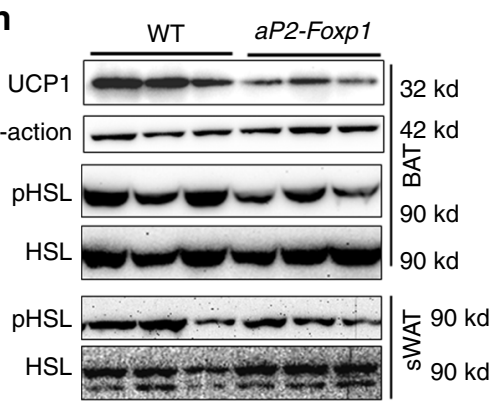

i

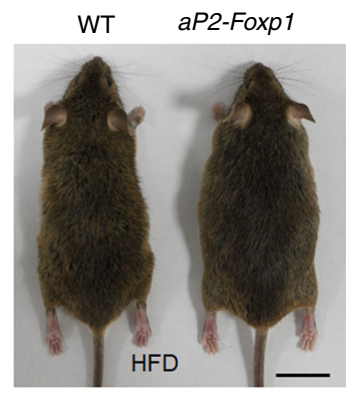

j

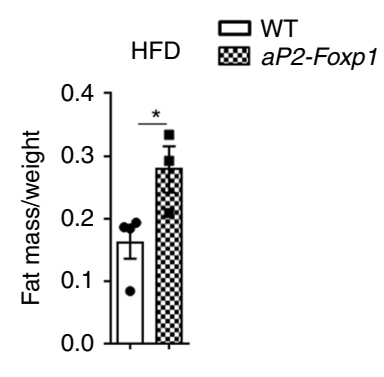

I

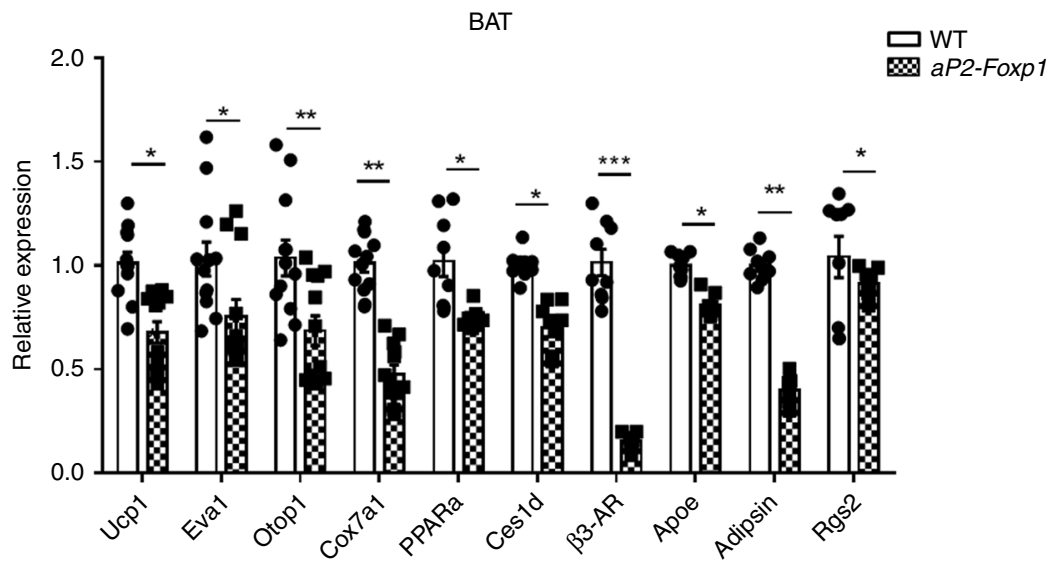

induction within BAT and sWAT (Fig. 4d, g, h) and less abundance of mitochondria (Fig. 4e, f). These observations suggest that overexpression of Foxpl disrupts the thermogenic program in adipose tissues.

Finally, our transgenic mice suffered perturbed lipolysis, as shown by their decreased phosphorylation of HSL (Fig. 4h)-a phenotype opposite to Foxp $1_{a P 2}{ }^{\Delta / \Delta}$ mutant mice (Supplementary Fig. 2j). As a consequence, the transgenic mice appeared to be sensitive to HFD-induced obesity (Fig. 4i, j). Collectively, these phenotypes were similar to those of previously reported mutant mice, such as BAT-specific Prdm16 knockout and global $\beta 3-A R$ knockout mice ${ }^{23,58}$. Taken together, the potency of adaptive 
Fig. 4 Overexpression of Foxp1 represses adaptive thermogenesis and is sensitive to HFD-induce obesity. a Representative bioluminescence imaging of two stains of aP2-Foxp1;Ucp1-Luc transgenic mice after 6-hour cold exposure. $n=3$ biologically independent mice/each group. Bar, $2 \mathrm{~cm}$. b Representative picture of BAT depot in aP2-Foxp1 transgenic mice. Bar, $0.5 \mathrm{~cm}$. c H\&E staining for adipose sections of BAT and sWAT from transgenic mice in (b). Bar, $10 \mu \mathrm{m}$. d IHC with UCP1 antibody for adipose sections from transgenic mice after 6 -h cold exposure. Bar, $10 \mu \mathrm{m}$. e Intracellular structure of adipocytes in BAT and sWAT from aP2-Foxp1 mice, as showed by transmission electronic microscope (TEM). Bar, $2 \mu \mathrm{m}$. f Assessment of mitochondria DNA abundance by qPCR for the BAT and sWAT from transgenic mice. $n=4$ biologically independent mice/each group. $\mathbf{g}$ qPCR analysis for Ucp1 expression in adipose tissues of transgenic mice. $n=4$ biologically independent samples. $\mathbf{h}$ Western blotting for UCP1, HSL, and phosphorylated HSL in BAT and sWAT. $\mathbf{i}$ Representative dorsal view of aP2-Foxp1 transgenic mice subjected to 8-week HFD feeding since at age of 3 months. Bar, $2 \mathrm{~cm}$. $\mathbf{j}$ Relative adiposity in transgenic mice (i). $n=4$ biologically independent mice/each group. $\mathbf{k}$ The heat map of relative expression of adipogenic genes in three strains of aP2Foxp1 transgenic mice at age of 3 weeks, as evaluated by RNA-seq. I qPCR analysis validated the BAT-selective genes expression in BAT from (k). $n=3$ biologically independent mice/each group; ${ }^{\star} P<0.05$; ${ }^{\star \star} P<0.01$; ${ }^{\star \star \star} P<0.001$; error bar, mean $\pm \mathrm{SEM}$

thermogenesis is impaired in aP2-Foxp1 transgenic mice in the opposite direction as $F o x p 1_{a P 2}{ }^{\Delta / \Delta}$ knockout mice.

To address molecular mechanisms utilized by Foxp1 in regulating the brown/beige program, we first compared gene expression profiles of BAT from 3-week-old wild type and aP2Foxp 1 mice by RNA-seq. As shown in the heat map of Fig. 4k, the expression levels of a set of BAT-selective genes (Ucp1, Eva1, $P P A R \alpha$, Otop1, Cox7a1, Ces1d) was down-regulated within the BAT of aP2-Foxp1 mice relative to controls. Next, we employed qPCR to validate the expression profiles of several marker genes in the BAT of transgenic mice. A significant decrease of $\beta 3-A R$ expression was observed among a broad panel of thermogenic genes (Fig. 4l). The changes in those gene expression profiles may underlie the defective thermogenesis in transgenic mice.

Foxp 1 directly represses $\beta 3$-AR transcription in adipocytes. $\beta 3$ $\mathrm{AR}$ is selectively expressed in the adipose tissues which mediate adaptive thermogenesis in responsive to adrenergic stimuli ${ }^{11,12}$. qPCR and western blotting analyses revealed a decrease in $\beta 3-\mathrm{AR}$ expression within the BAT and sWAT of aP2-Foxp1 transgenic mice (Fig. 5a, b), and reciprocally, an increase of $\beta 3$-AR expression in Foxp $1_{a P 2}{ }^{\Delta / \Delta}$ knockout mice (Fig. $5 c, d$ ). These data suggested that alteration of $\beta 3$-AR expression may partially account for the disturbance of thermogenesis in both Foxp1 knockout and transgenic mice. Of note, a consensus Foxp1 binding site (TTATTTAT) was detected at $-2251 \mathrm{bp}$ upstream of the $\beta 3-A R$ promoter (Fig. 5f). This led us to conduct promoter occupancy analysis by ChIP-seq and ChIP-PCR based on SVF progenitor cells, confirming this site within the chromatin of the $\beta 3-A R$ promoter (Fig. 5e, f). In addition, the increased OCR of SVFderived brown adipocytes in Foxp $1_{a P 2}{ }^{\Delta / \Delta}$ knockout mice (Supplementary Fig. 3i), was arrested either by exposure to the $\beta 3-\mathrm{AR}$ antagonist L748337, or by knockdown with $\beta 3$ - $A R$-shRNA (Fig. 5g). Infrared imaging revealed a decline of dorsal skin temperature in aP2-Foxp1 transgenic mice (Fig. 5h). Normal temperature was restored when the mutant mice were challenged by the $\beta 3$-AR agonist CL-316,243 (Fig. 5i). Thus, the $\beta 3-A R$ agonist could rescue the thermogenic defects of the transgenic mice (Fig. 5h), whereas $\beta 3-\mathrm{AR}$ antagonist could block the enhanced thermogenic phenotypes observed in the knockout mice (Fig. 5g). Furthermore, we observed that brown adipocytes derived from SVF of $F o x p 1_{a P 2}{ }^{\Delta / \Delta}$ knockout and aP2-Foxp1 transgenic mice altered the desensitization behavior of $\beta 3-A R$, as evidenced by the $\beta 3$-AR expression profiles during 6-hours treatment with CL-316,243. As shown in Fig. 5j, the downregulation of $\beta 3-A R$ in brown adipocytes from $\operatorname{Foxp}_{a P 2} \Delta / \Delta$ mice declined sharply as compared to the wild type controls, whereas those of transgenic mice declined more smoothly. Together these findings suggest that Foxp1, at least in partial, controls thermogenesis through repressing of $\beta 3$-AR transcription within brown/ beige adipocytes.
Foxp1 forms a complex with Prdm16-C/ebp $\beta$ proteins. We previously observed that Foxp1 regulates the adipogenic potential of mesenchymal stem cells by interacting with the $\mathrm{C} / \mathrm{ebp} \beta / \delta$ complex ${ }^{49}$. Both $\mathrm{C} / \mathrm{ebp} \beta$ and Prdm16 are critical regulators of brown adipocyte differentiation and thermogenesis ${ }^{34}$. Here, we observed via co-immunoprecipitation (Co-IP) assays that Foxp1 interacted with $\mathrm{C} / \mathrm{ebp} \beta$ and Prdm16 in SVF cells isolated from BAT (Fig. 6a). We validated these results in HEK293T cells cotransfected with Foxp1-His, Prdm16-Flag and C/ebp $\beta-M y c$ expression plasmids (Fig. 6b). In support, immunofluorescence analysis also detected co-localization of Foxp1 and Prdm16 within the nucleus of brown adipocytes (Fig. 6c). Luciferase reporter assays employing a PPAR $y$ promoter-driven luciferase vector further showed that Foxp1 repressed transactivation ability of Prdm16 in 3T3-L1 cells (Fig. 6d). Similarly, assays employing a $\beta 3-A R$ promoter-driven luciferase vector revealed that Foxp1 repressed transcription of the $\beta 3-A R$ by antagonizing the transactivation ability of the Prdm16-C/ebp $\beta$ complex (Fig.6e). Collectively, these data suggest a mechanism by which Foxp1 forms a complex with Prdm16-C/ebp $\beta$ proteins to repress $\beta 3-A R$ transcription (Fig. 6f).

\section{Discussion}

In mammals, brown/beige thermogenesis is triggered to dissipate heat for thermoregulation upon cold challenge, mainly through a thermogenic program mediated by $\beta$-AR signaling ${ }^{59}$. Thus, the activity of $\beta$-AR signaling has to be precisely controlled. In pheochromocytoma (PHEO) patients, WAT browning was detected as a result of severe adrenergic stress under continuously high levels of catecholamines, which disrupted the energy balance in adipose tissues to result in weight $\operatorname{loss}^{51}$. To restrain this overactivation of BAT thermogenic program, a number of transcriptional co-repressors has been employed to counteract the activity of PGC-1a, e.g. Rip 140 and Twist $1^{36,37}$, or to repress transcription of $U c p 1$, e.g. $\mathrm{Rb}^{60}$ and $\mathrm{LXRa}^{61}$. In this study, we examined the influence of Foxp1 on thermogenesis in adipocytes from knockout mice employing Myf5-Cre, aP2-Cre and Adiponectin-Cre, as well as by transgenic mice with Foxp1 overexpression in adipocytes. Our analyses identify Foxp1 as a repressor for brown/beige adipocyte differentiation and energy expenditure. Luciferase reporter suggested that Foxp1B, the isoform that was expressed in BAT and sWAT, mostly accounted for the repressive function in BAT activation and thermogenesis (Supplementary Fig. 5c). We further show that Foxp1 expression could be induced by a $\beta 3-\mathrm{AR} / \mathrm{cAMP} /$ Erk $1 / 2$ cascade. As a negative feedback brake, Foxp1 acts to repress thermogenesis through antagonizing the action of the Prdm16-C/ebp $\beta$ complex to repress $\beta 3$-AR transcription (Fig. 6f). We suggest that this circuit may precisely control $\beta 3-\mathrm{AR}$ transcription to maintain the setpoint of body weight.

According to the robust phenotypes in the four categories of mutant mice, Foxp1 performs a consistent function in the 
a

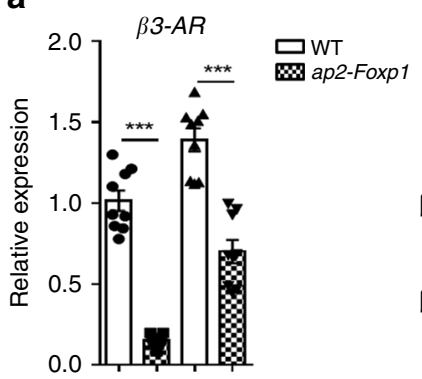

e

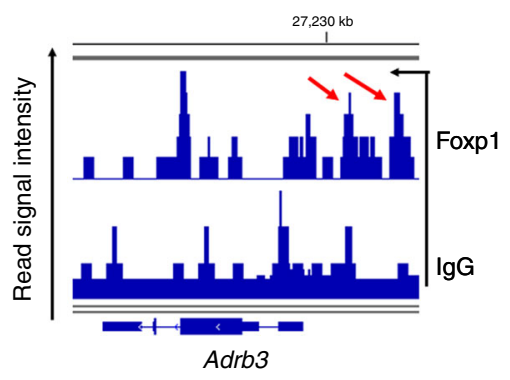

h

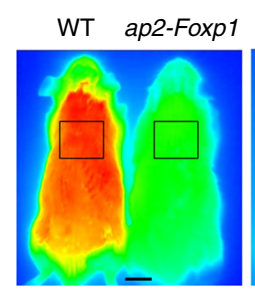

b

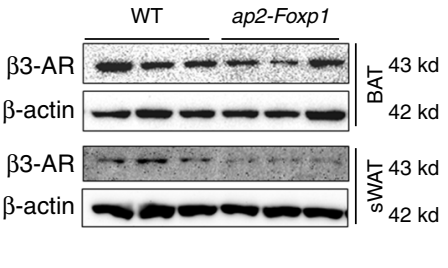

c

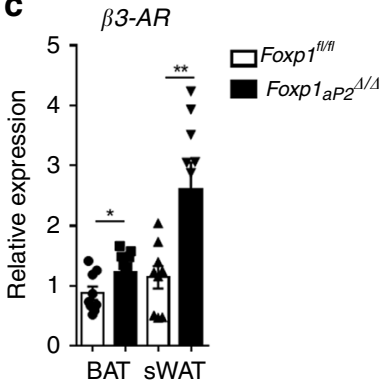

d

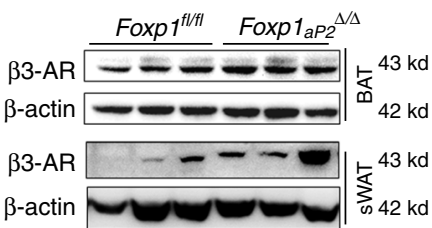

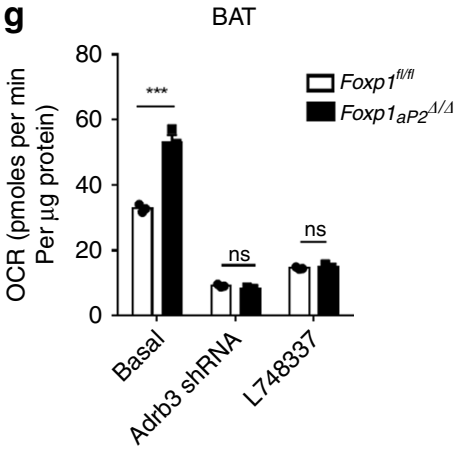

f -2251 bp TTATTTAT $\longrightarrow$
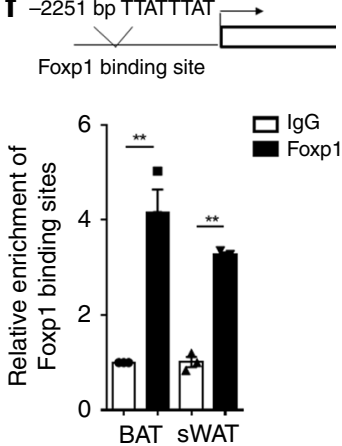

i
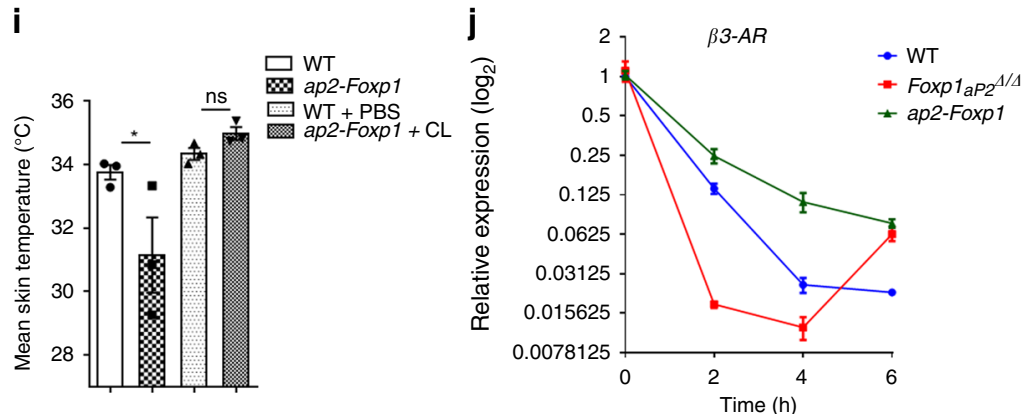

Fig. 5 Foxp1 represses $\beta 3-A R$ transcription in adipocytes. $\mathbf{a}, \mathbf{b} \beta 3-\mathrm{AR}$ expression in BAT and sWAT were assessed by qPCR and western blotting in aP2Foxp 1 transgenic mice at 2 month old. $n=3$ biologically independent samples/each group. $\mathbf{c}, \mathbf{d} \beta 3$-AR expression in BAT and sWAT were assessed by qPCR and western blotting in Foxp $1_{a P 2}{ }^{\Delta / \Delta}$ knockout mice at 2 months old. $n=3$ biologically independent samples/each group. e ChIP-seq profile showed the Foxp1 binding sites within Adrb3 gene promoter region. Red arrows indicated the potential Foxp1 binding sites. Adrb3 gene was reversely transcribed. ChIP was based on SVF cells derived from BAT. f Upper panel showed the location of Foxp1 binding site at -2251 bp upstream of $\beta 3-A R$ gene promoter with a schematic drawing. ChIP-PCR confirmed the relative enrichment of Foxp1 binding sites (lower panel). $n=3$ biologically independent samples/each group. g Basic OCR of SVF-derived brown adipocytes from Foxp $1_{a P 2} \Delta / \Delta$ mice, which were transfected $\beta 3$-AR-shRNA lentivirus or administrated with $\beta 3$-AR inhibitor $\mathrm{L} 748337(10 \mu \mathrm{M})$ for $1 \mathrm{~h}$. $n=3$ biologically independent experiments. h Representative infrared imaging of aP2-Foxp1 transgenic mice with or without 6-hour CL-316,243 (10 $\mu \mathrm{M}$ ) exposure. Bar, $1 \mathrm{~cm}$. i Quantification of average skin temperature in boxed regions in (h). $n=4$ biologically independent mice/each group. Samples were isolated from mice at age of 2 months. $\mathbf{j}$ CL-stimulated $\beta 3$-AR desensitization in SVF-derived brown adipocytes from aP2-Foxp1 transgenic mice and Foxp $1_{a P 2} \Delta / \Delta$ knockout mice. $\beta 3-A R$ expression was assessed by qPCR during the 6 -h time course of CL-316243 $(0.1 \mu \mathrm{M})$ treatment. $n=3$ biologically independent samples/each group. ${ }^{\star} P<0.05 ;{ }^{\star \star} P<0.01$; ${ }^{\star \star \star} P<0.001$; error bar, mean \pm SEM

multiple steps of adipocyte differentiation and metabolism. Depletion of Foxp1 via Myf5-Cre or Ad-Cre in adipocytes potentiated brown or beige adipocyte differentiation under mildly ambient temperature (Fig. 2). We previously observed that Foxp1 suppresses adipogenic commitment of mesenchymal stem cells by repressing PPARy transcription ${ }^{49}$. Here we show that Foxp1 similarly regulates early commitment/differentiation of brown adipocytes from progenitor cells (Fig. 2a-d), through repressing the transactivation ability of $\operatorname{Prdm} 16-\mathrm{C} / \mathrm{ebp} \beta$, two key regulators of brown or beige adipocyte differentiation ${ }^{4,62}$. Similar defects in thermogenesis and energy expenditure were observed in both Foxp $1_{a P 2}{ }^{\Delta / \Delta}$ and Foxp $1_{A d} \Delta / \Delta$ mice. Of note, we noticed that defects in adaptive thermogenesis in $F o x p 1_{a P 2} \Delta / \Delta$ mice were much more penetrant than that in $\operatorname{Foxp}_{A d} \Delta / \Delta$ mice at comparable ages. White adipose tissue browning was only evident in
Foxp $1_{A d} \Delta / \Delta$ mice under several-hours cold challenge (Fig. $2 \mathrm{~h}$ ). Elevated energy expenditure also was considerably more pronounced in Foxp $1_{a P 2}{ }^{\Delta / \Delta}$ mice than in Foxp $1_{A d} \Delta / \Delta$ mice (Fig. 3 and Supplementary Fig. 4). This discrepancy in thermogenic defects may result from differences in the timing and specificity of Cre activities of the two strains; i.e., the latter occurred earlier than the former as previously suggested ${ }^{63}$. Interestingly, Foxp1 regulates liver glycogenesis ${ }^{44}$, thus the impact of Foxp1 on glucose homeostasis, which remains to be tested in adipocytes, may also underpin its regulation of energy metabolism described here.

Distinct subtypes of $\beta$-ARs have been shown to possess different properties of desensitization within different tissues. For example, the agonist-stimulated desensitization of $\beta 1$ or $\beta 2-\mathrm{AR}$ is fulfilled by $\beta$-Arrestin-dependent sequestration through receptor 
a

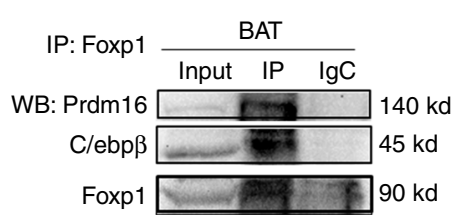

b

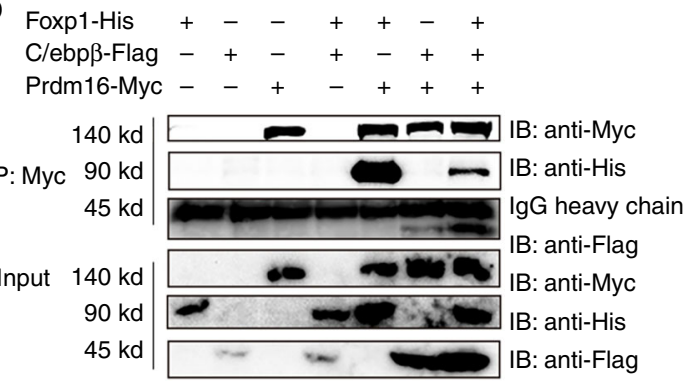

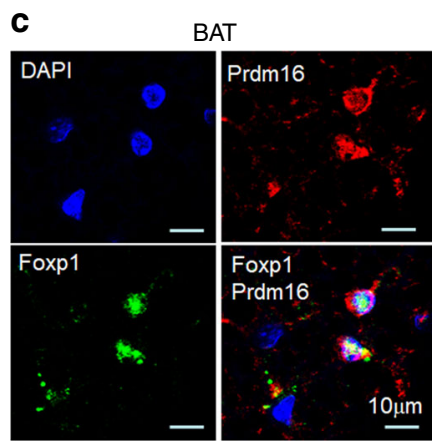
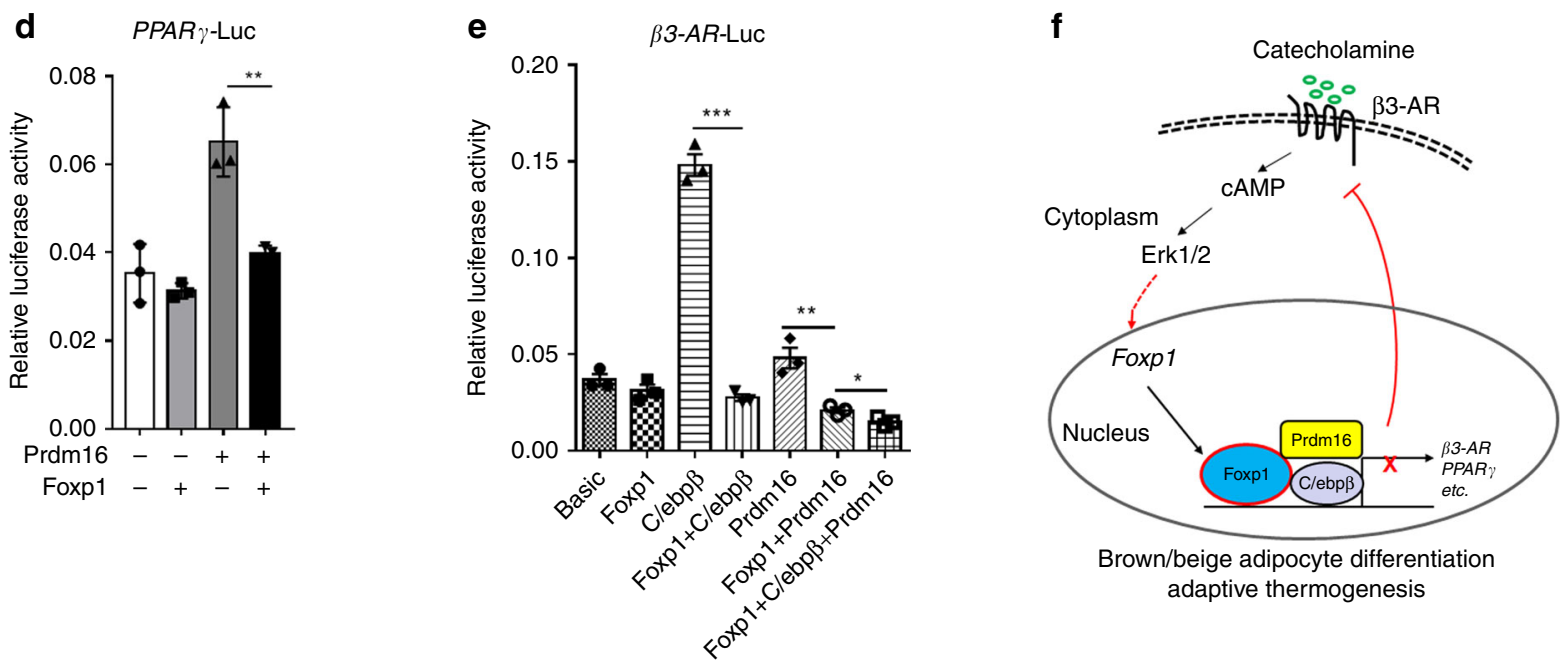

Fig. 6 Foxp1 physically interacts with Prdm16-C/ebp $\beta$ complex in brown adipocytes. a, b Co-immunoprecipitation validated the physical interaction between Foxp1, C/ebp $\beta$ and Prdm16 proteins in cell lysates from 293T cell lines transfected with indicated plasmids (a), or brown adipocytes induced from stromal vascular fractions (b). c Immunofluorescence analysis showed the co-localization of Foxp1 and Prdm16 in the nucleus of BAT from wild type mice at age of 1 month old. Bar, $10 \mu \mathrm{m}$. d Luciferase reporter assay showed the transactivation of PPAR $\gamma$-Luc by Foxp1 and Prdm16 protein in 3T3-L1 cell lines. $n=$ 3 biologically independent samples/each group. e Luciferase reporter assay showed the transactivation of $\beta 3$ - $A R$-Luc by Foxp1, C/ebp $\beta$ and Prdm16 protein in 293 T cell lines. $n=3$ biologically independent experiments; ${ }^{\star} P<0.05,{ }^{\star \star} P<0.01,{ }^{\star \star \star} P<0.001$; error bar, mean \pm SEM. f Diagram depicting the mechanisms that Foxp1 represses brown/beige adipocyte differentiation and adaptive thermogenesis. Expression of Foxp1 is induced by $\beta 3-A R / c A M P /$ Erk1/2 cascades. Conversely, Foxp1 suppresses $\beta 3-A R$ transcription by counteracting the activity of Prdm16-C/ebp $\beta$ complex

phosphorylation by $\mathrm{GRK}^{29,64}$. In contrast, $\beta 3$-AR displays resistance to typical desensitization due to a lack of putative GRK phosphorylation sites ${ }^{65,66}$. Instead, $\beta 3$-AR may adopt a desensitization strategy primarily based on decreasing transcriptional expression in adipocytes ${ }^{25-28}$. Our findings may provide a clue toward understanding the mechanisms underlying the unique strategy of $\beta 3-\mathrm{AR}$ desensitization. Adrenergic stimuli swiftly induced Foxp1 mRNA and protein level in adipocytes, which conversely decreased $\beta 3$-AR mRNA levels. As showed in Fig. $6 \mathrm{j}$, alterations of Foxpl abundance profoundly affected the sensitivity of $\beta 3$-AR desensitization. Of note, it has to be kept in mind that the $\beta 3$-AR pathway only partially account for the function of Foxp1 in BAT activation and thermogenesis, given that Foxp1deficient cells exhibit higher cell-autonomous ability to differentiate into brown adipocytes or beige adipocytes in cultured conditions without $\beta 3$-AR agonist stimulation. Recently, a new type of glycolytic beige adipocytes, which are $\beta$-AR independent and derived from $\mathrm{MyoD}^{+}$progenitor cells, is characterized in WAT upon prolonged cold exposure ${ }^{67}$. Surprisingly, we also observed upregulation of glycolytic beige-related marker genes $(M y o D, M y h 1, G A B P \alpha)$ in Foxp $1_{a P 2}{ }^{\Delta / \Delta}$ WAT (Supplementary Fig. 6a). These findings suggest that Foxp1 may also regulate glycolytic beige adipocyte activation through a $\beta$-AR independent manner. ChIP-seq analysis also suggested that Foxp1 may exert extensive influence on BAT energy metabolism through regulation of theromogenic genes including $P P A R \gamma, P G C-1 \alpha$, Twist1, Dio2, Irx3 etc (Supplementary Table 1).

Of note, alterations between $\beta 3-\mathrm{AR}$ mRNA and protein levels are not synchronized within a short term during the desensitization process. For instance, when SVF-derived brown/beige adipocytes were stimulated by a $\beta 3-\mathrm{AR}$ agonist for $8 \mathrm{~h}$, we detected changes in $\beta 3$-AR mRNA (Fig. 1e-g), but not in protein levels (Supplementary Fig. 6b). In addition, $\beta 3$-AR agonists have been promising pharmaceutical targets for a number of metabolic diseases. For instance, Mirabegron is a $\beta 3-\mathrm{AR}$ agonist that has been approved in humans to treat overactive bladder syndrome ${ }^{68}$. However, $\beta 3$-AR agonists still lack efficacy for combating human obesity. This may, in part, owe to the undesirable side effects of $\beta 3-A R$ desensitization. We submit that further investigation into mechanisms underlying Foxp1-mediated $\beta 3$-AR regulation will cast new light upon the development of therapeutic strategies for obesity.

\section{Methods}

Mice. The Foxp1 $1^{f l f l}$ has been used in our previous studies ${ }^{69}$. aP2-Cre (Stock no. 005069 in Jax Lab), Adiponectin-Cre (Stock no. 028020), Thermo Mouse (Stock no. 026690) were obtained from Jax lab. Myf5-Cre mice was kindly provided by Prof. Dahai Zhu in Peking Union Medical College. Transgenic vector containing $5.4 \mathrm{~kb}$ promoter of Fabp4 (aP2) gene were kindly provided by Prof. Qiqun Tang in Fudan University. The genetic backgrounds of all knockout mice were $\mathrm{C} 57 \mathrm{Bl} / 6 \mathrm{~J}$ and the background of transgenic mice were $129 \mathrm{~S} 1 / \mathrm{Sv}$. Mice were bred with standard 
rodent chow food or HFD. Male mice were used in the experiments unless otherwise indicated. The experiments were not randomized, and the investigators were not blinded to allocation during experiments or outcome assessments. All animal experiments were performed according to the guidelines (SYXK 2011-0112) and received ethical approval from committee of Bio-X Institutes of Shanghai Jiao Tong University.

Human adipose tissue samples. Human browning omental adipose tissue for immunohistochemistry was obtained from clinically and pathologically diagnosed patients with pheochromocytoma and sex-, age-, and BMI-matched control subjects. Three samples were analyzed with immunofluorescence or IHC in this study. Primary human stromal vascular fraction (SVF) from subcutaneous WAT (sWAT) for the beige adipocyte induction were obtained from underaged subjects. The adipose tissue pieces were obtained during surgical procedures followed by immediate storage in liquid nitrogen and fixation in formalin. The human study was performed in accordance with relevant guidelines and received ethical approval from the Institutional Review Board of Ruijin Hospital, Shanghai Jiao Tong University School of Medicine. Written informed consent was provided from each participant prior to inclusion in the study.

Metabolic Study, bioluminescence, and infrared imaging. Minispec TD-NMR Analysers (Bruker Instruments) were used to evaluate adiposity composition on anesthetized animals. Food intake, energy expenditure, $\mathrm{O}_{2}$ consumption, $\mathrm{CO}_{2}$ production and physical activity were measured by using indirect calorimetry system (Oxymax, Columbus Instruments), installed under a constant environmental temperature $\left(22^{\circ} \mathrm{C}\right)$ and a 12 -h light $(07: 00-19: 00 \mathrm{~h}), 12$-h dark cycle (19:00-07:00 h). Mice in each chamber had free access to food and water. The raw data were normalized by body weight and the histograms of day (07:00-19:00 h) and night (19:00-07:00 h) values were the mean value of all points measured during the 12-h period. Bioluminescence analysis for ThermoMouse is performed using Lumina III (Perkin Elmer, IVIS). An infrared camera (T650sc, emissivity of 0.98 , FLiR Systems) is placed over top of the anesthetized mouse to acquire static image according to standard protocols.

Glucose tolerance test (GTT) and insulin tolerance test (ITT). For GTT, mice were given i.p. injection of $100 \mathrm{mg} / \mathrm{ml} \mathrm{D}$-glucose $(2 \mathrm{~g} / \mathrm{kg}$ body weight) after overnight fasting, and tail blood glucose concentrations were measured by a glucometer (AccuCheck Active, Roche). For ITT, mice were fasted for 4 h before i.p. administration of human insulin (Santa Cruz) $(0.75 \mathrm{U} / \mathrm{kg}$ body weight), and tail blood glucose concentrations were monitored.

Immunohistochemistry, immunofluorescence, and TEM. Adipose tissues were fixed in $4 \%$ PFA for $24 \mathrm{~h}$ at $4{ }^{\circ} \mathrm{C}$, embedded in paraffin or tissue freezing medium (Leica) and sectioned to $8 \mu \mathrm{m}$. HE staining was conducted according to standard protocols. For immunofluorescence, heat-induced antigen retrieval with sodium citrate buffer ( $10 \mathrm{mM}$ sodium citrate, $0.05 \%$ Tween $20, \mathrm{pH} 6.0$ ) was performed before bone sections were blocked with $10 \%$ normal serum containing $1 \% \mathrm{BSA}$ in TBST ( $\mathrm{pH}$ 7.6) for $2 \mathrm{~h}$ at room temperature, then incubated overnight at $4{ }^{\circ} \mathrm{C}$ with primary antibodies to mouse Foxp1 (Millipore, ABE68, 1:100), Ucp1 (Abcam, ab10893, 1:50), Prdm16 (Abcam, ab106410, 1:50). Subsequently, sections were incubated with secondary fluorescent-conjugated or HRP-conjugated antibodies at room temperature for $2 \mathrm{~h}$ in the dark. Samples were imaged by the Leica TCS SP5 confocal microscope, Leica DM2500, or Leica 3000B microscope. Transmission electron microscopy (TEM) of white and brown adipose tissue was carried out in accordance with a previous study.

Cell cultures. For SVF cell isolation, primary BAT and sWAT were digested with $1 \mathrm{mg} \mathrm{ml}^{-1}$ collagenase type I (Sigma) in DMEM (Invitrogen) supplemented with $1 \%$ bovine serum albumin for $25 \mathrm{~min}$ at $37^{\circ} \mathrm{C}$, followed by density separation. The digestions were quenched with DMEM containing 10\% FBS, and filtered through 70 -mm filters to remove connective tissues and undigested trunks of tissues. Cells were then centrifuged at $1000 \times g$ for $5 \mathrm{~min}$ to separate the SVF cells in the floating layer. The freshly isolated SVF cells were seeded and cultured in growth medium containing DMEM, 20\% FBS, $1 \%$ penicillin/streptomycin $(\mathrm{P} / \mathrm{S})$ at $37^{\circ} \mathrm{C}$ with $5 \%$ $\mathrm{CO}_{2}$ for 3 days, followed by feeding with fresh medium every 2 days to reach confluence. For brown adipocyte differentiation, the cells were induced with induction medium contains DMEM, $10 \% \mathrm{FBS}, 5 \mu \mathrm{g} \mathrm{ml}{ }^{-1}$ insulin, $0.5 \mathrm{mM}$ isobutylmethylxanthine (Sigma), $1 \mu \mathrm{M}$ dexamethasone (Sigma), $50 \mathrm{nM}$ T3 (Sigma) and $5 \mu \mathrm{M}$ troglitazone (Sigma) for $48 \mathrm{~h}$, and further in growth medium supplemented with insulin, T3 and troglitazone for six days followed by $0.5 \mathrm{mM}$ cyclic AMP (Sigma) treatment for another 4 h. 3T3-L1 (ATCC) and HEK293T (ATCC) were cultured in DMEM with 10\% FBS. For 3T3-L1 adipogenic differentiation, cells of $100 \%$ confluence were kept in growth medium for 2 days then induced with induction medium for 2 days, after that differentiated in differentiation medium (without T3) for 6 days. For oil red staining, cultured cells were washed with PBS and fixed with $10 \%$ formaldehyde for $15 \mathrm{~min}$ at room temperature. Then the cells were stained using the Oil red $\mathrm{O}$ working solutions ( $5 \mathrm{~g} / \mathrm{l}$ in isopropanol) and $4 \mathrm{ml}$ $\mathrm{ddH}_{2} \mathrm{O}$ for $30 \mathrm{~min}$. After staining, the cells were washed with $60 \%$ isopropanol and pictured.
Adipocyte OCR measurement. Primary SVF cells from BAT and sWAT were isolated and cultured for 3 days before being plated in XF cell culture microplates (Seahorse Bioscience). SVF cells (10,000 cells) were seeded in each well, and each treatment included cells from three BAT or sWAT replicates. After 6-day differentiation, cultured adipocytes were washed twice and pre-incubated in XF medium (supplemented with $25 \mathrm{mM}$ glucose, $2 \mathrm{mM}$ glutamine and $1 \mathrm{mM}$ pyruvate) for $1-2 \mathrm{~h}$ at $37^{\circ} \mathrm{C}$ without $\mathrm{CO}_{2}$. The OCR was measured using the XF Extracellular Flux Analyser (Seahorse Biosciences). Oligomycin (2 mM), FCCP (2 mM), and Antimycin A ( $0.5 \mathrm{mM})$ were preloaded into cartridges and injected into XF wells in succession. OCR was calculated as a function of time (pmoles per minute per $\mu \mathrm{g}$ protein).

shRNA lentivirus preparation. To construct shRNA for Foxp1-shRNA or $\beta 3$-AR shRNA virus, the forward and reverse oligos for each shRNA were mixed and denatured at $95^{\circ} \mathrm{C}$ for $5 \mathrm{~min}$, annealed at $58^{\circ} \mathrm{C}$ for $10 \mathrm{~min}$, and then digested by $\mathrm{XbaI}$ and BamHI before cloning into lentivirus-based vector pLenti-shRNA-NF1 GFP. The lentivirus were packaged in 293T cells with helper vector pMD2G and psPAX2 by transfecting cells with indicated constructs. After 48-h culture, supernatant was collected by centrifuge at $1000 \times g$ for $5 \mathrm{~min}$, followed by filtered with MILLEX GP. The oligos for shRNA construct were listed in Supplementary Table 2 .

ChIP-seq analysis and Co-IP. SVF cells were isolated from mouse BAT or sWAT and expanded in passaging cultures for 2 weeks. The ChIP samples were prepared as following ${ }^{70}$. Briefly, 2-5 million cells were cross-linked with $1 \%$ formaldehyde (Sigma-Aldrich) for $10 \mathrm{~min}$ at room temperature, and then incubated with $125 \mathrm{mM}$ glycine for $5 \mathrm{~min}$ to quench the cross-linking reaction. The harvested cells were sonicated on ice to generate DNA fragments of 200-500 bp. The fragmented chromatin fragments were immunoprecipitated with $20 \mu \mathrm{l}$ of protein A/G magnetic beads (Millipore) coupled with $4 \mu \mathrm{g}$ of anti-Foxp1 antibody (Millipore, ABE68, $1: 200)$ at $4{ }^{\circ} \mathrm{C}$ overnight with rotation. ChIP DNA was treated with protein $\mathrm{K}$ (Thermo Fisher) at $55^{\circ} \mathrm{C}$ for $6.5 \mathrm{~h}$ for decrosslinking. Both ChIP and input DNA libraries were generated using the NEBNext Ultra II DNA Library Prep Kit for Illumina (E7645, NEB). The DNA libraries were amplified for 18 cycles and subjected to sequencing by Illumina sequencer. For ChIP-PCR, purified DNA was quantified using quantitative PCR. The primer sequences are listed in Supplementary Table 2 .

For in vitro co-immunoprecipitation (Co-IP), His- or FLAG-tagged proteins were produced in HEK293T transfected by FuGENE with corresponding plasmids. For in vivo Co-IP, SVF cells were isolated and cultured from wild-type mice. Total cell lysates were incubated overnight at $4{ }^{\circ} \mathrm{C}$ with antibodies or normal IgG (Santa Cruz) as control. Antibody-antigen complexes were absorbed by Protein A/G PLUS-Agarose (Santa Cruz, sc-2003). After several washes, samples were boiled and analyzed by western blotting.

qPCR and western blotting. Total RNA was extracted with Trizol (Invitrogen), reverse transcriptase was used for CDNA generation with the GoScript reverse transcription system (Promega). qPCR was performed with a real time PCR system (ABI 7500) using SYBR Green (Roche). The primer sequences are listed in Supplementary Table 2. For western blotting, cells were lysed and protein samples were incubated with primary antibodies against Foxp1 (Millipore, ABE68, 1:1000), C/ $\operatorname{ebp} \beta$ (Santa Cruz, sc-150, 1:500), UCP1(Abcam, ab10893, 1:1000), PGC-1 $\alpha$ (Milipore, ab3242 1:1000), p38 (CST, 9212s, 1:1000), phosphorylated p38 (CST, 9211s, 1:1000), HSL (CST, 4107, 1:1000) and phosphorylated HSL (CST, 4126, 1:1000), $\beta 3$-AR (mybioscience, MBS253490, 1:1000), His-Tag (MBL, M136-3, 1:2000), FLAG (Agilent, 200471, 1:2000) or $\beta$-actin (Selleck, A1016,1:2000) at $4{ }^{\circ} \mathrm{C}$ overnight. Proteins were visualized using HRP-conjugated secondary antibody and chemiluminescent HRP substrate (Millipore).

Luciferase assay. Luciferase assays were performed in HEK293T or 3T3-L1 cells The reporter plasmid, PPAR $\gamma$-Luc containing a $2.2 \mathrm{~kb}$ fragment of the 5' flanking region of the PPAR $\gamma$ gene, was obtained from Dr. Hiroshi Takayanagi of the Tokyo Medical and Dental University. Adrb3-Luc plasmid containing $4.3 \mathrm{~kb}$ of the 5 ' flanking region of $A d r b 3$ gene, was constructed by our lab. The expression plasmids of Foxp1 and $\operatorname{Cebp} \beta$ were constructed into the pcDNA3.0 vector. Cells were transfected using FuGENE HD (Promega) in 24-well plates. The transfection amount of each plasmid was $200 \mathrm{ng}$, and the total amount of transfected DNA across each transfection was balanced by pcDNA3.0 plasmids when necessary. After $32 \mathrm{~h}$, dual luciferase assay was performed according to the manufacturer's protocols (Promega).

Statistical analysis. All data are presented as mean \pm SEM. Error bars are SEM. Two-tailed Student's $t$-tests for comparisons between two groups. For all experiments, $P \geq 0.05$ were marked as ns, $P<0.05$ were considered significant and indicated by ${ }^{\star} P<0.01$ were indicated by ${ }^{* *} P<0.001$ were indicated by ${ }^{* * *}$.

Reporting summary. Further information on research design is available in the Nature Research Reporting Summary linked to this article. 


\section{Data availability}

All raw western blotting data could be found in the Source Data. Raw ChIP-seq data could be available in NCBI (SRA) database (accessions no: PRJNA547458). All relevant data are available from the authors.

Received: 20 September 2018; Accepted: 2 October 2019; Published online: 07 November 2019

\section{References}

1. Rosen, E. D. \& Spiegelman, B. M. What we talk about when we talk about fat. Cell 156, 20-44 (2014).

2. Gesta, S., Tseng, Y. H. \& Kahn, C. R. Developmental origin of fat: tracking obesity to its source. Cell 131, 242-256 (2007).

3. Frontini, A. \& Cinti, S. Distribution and development of brown adipocytes in the murine and human adipose organ. Cell Metab. 11, 253-256 (2010).

4. Seale, P. et al. PRDM16 controls a brown fat/skeletal muscle switch. Nature 454, 961-967 (2008).

5. $\mathrm{Wu}, \mathrm{J}$. et al. Beige adipocytes are a distinct type of thermogenic fat cell in mouse and human. Cell 150, 366-376 (2012).

6. Wu, J., Cohen, P. \& Spiegelman, B. M. Adaptive thermogenesis in adipocytes: is beige the new brown? Genes Dev. 27, 234-250 (2013).

7. Cao, L. et al. White to brown fat phenotypic switch induced by genetic and environmental activation of a hypothalamic-adipocyte axis. Cell Metab. 14, 324-338 (2011).

8. Sidossis, L. S. et al. Browning of subcutaneous white adipose tissue in humans after severe adrenergic stress. Cell Metab. 22, 219-227 (2015).

9. Arner, P. et al. Dynamics of human adipose lipid turnover in health and metabolic disease. Nature 478, 110-113 (2011).

10. Harms, M. \& Seale, P. Brown and beige fat: development, function and therapeutic potential. Nat. Med 19, 1252-1263 (2013).

11. Jimenez, M. et al. $\operatorname{Beta}(1) / \operatorname{beta}(2) /$ beta(3)-adrenoceptor knockout mice are obese and cold-sensitive but have normal lipolytic responses to fasting. FEBS Lett. 530, 37-40 (2002).

12. Bachman, E. S. et al. betaAR signaling required for diet-induced thermogenesis and obesity resistance. Science 297, 843-845 (2002).

13. Robidoux, J., Martin, T. L. \& Collins, S. Beta-adrenergic receptors and regulation of energy expenditure: a family affair. Annu Rev. Pharm. Toxicol. 44, 297-323 (2004).

14. Inagaki, T., Sakai, J. \& Kajimura, S. Transcriptional and epigenetic control of brown and beige adipose cell fate and function. Nat. Rev. Mol. Cell Biol. 17, 480-495 (2016).

15. Greenberg, A. S. et al. Stimulation of lipolysis and hormone-sensitive lipase via the extracellular signal-regulated kinase pathway. J. Biol. Chem. 276, 45456-45461 (2001).

16. Robidoux, J. et al. Maximal beta3-adrenergic regulation of lipolysis involves Src and epidermal growth factor receptor-dependent ERK1/2 activation. J. Biol. Chem. 281, 37794-37802 (2006).

17. Jiang, Y., Berry, D. C. \& Graff, J. M. Distinct cellular and molecular mechanisms for beta3 adrenergic receptor-induced beige adipocyte formation. eLife 6, e30329 (2017).

18. Ghorbani, M., Teimourian, S., Farzad, R. \& Asl, N. N. Apparent histological changes of adipocytes after treatment with CL 316,243, a beta-3-adrenergic receptor agonist. Drug Des., Dev. Ther. 9, 669-676 (2015).

19. Cernecka, H., Sand, C. \& Michel, M. C. The odd sibling: features of beta3adrenoceptor pharmacology. Mol. Pharm. 86, 479-484 (2014).

20. Yoshida, T. et al. Mutation of beta 3-adrenergic-receptor gene and response to treatment of obesity. Lancet 346, 1433-1434 (1995).

21. Widen, E. et al. Association of a polymorphism in the beta 3-adrenergicreceptor gene with features of the insulin resistance syndrome in Finns. New Engl. J. Med. 333, 348-351 (1995).

22. Walston, J. et al. Time of onset of non-insulin-dependent diabetes mellitus and genetic variation in the beta 3-adrenergic-receptor gene. New Engl. J. Med. 333, 343-347 (1995).

23. Susulic, V. S. et al. Targeted disruption of the beta 3-adrenergic receptor gene. J. Biol. Chem. 270, 29483-29492 (1995).

24. Collins, S. et al. Impaired expression and functional activity of the beta 3- and beta 1-adrenergic receptors in adipose tissue of congenitally obese (C57BL/6 J ob/ob) mice. Mol. Endocrinol. 8, 518-527 (1994).

25. Granneman, J. G. \& Lahners, K. N. Differential adrenergic regulation of beta 1- and beta 3-adrenoreceptor messenger ribonucleic acids in adipose tissues. Endocrinology 130, 109-114 (1992).

26. Bengtsson, T., Redegren, K., Strosberg, A. D., Nedergaard, J. \& Cannon, B Down-regulation of beta3 adrenoreceptor gene expression in brown fat cells is transient and recovery is dependent upon a short-lived protein factor. J. Biol. Chem. 271, 33366-33375 (1996).
27. Scarpace, P. J., Matheny, M. \& Tumer, N. Differential down-regulation of beta3-adrenergic receptor mRNA and signal transduction by cold exposure in brown adipose tissue of young and senescent rats. Pflug. Arch. 437, 479-483 (1999).

28. Bengtsson, T., Cannon, B. \& Nedergaard, J. Differential adrenergic regulation of the gene expression of the beta-adrenoceptor subtypes betal, beta 2 and beta3 in brown adipocytes. Biochem J. 347(Pt 3), 643-651 (2000).

29. Luttrell, L. M. et al. Beta-arrestin-dependent formation of beta2 adrenergic receptor-Src protein kinase complexes. Science 283, 655-661 (1999).

30. Kajimura, S., Seale, P. \& Spiegelman, B. M. Transcriptional control of brown fat development. Cell Metab. 11, 257-262 (2010).

31. Seale, P., Kajimura, S. \& Spiegelman, B. M. Transcriptional control of brown adipocyte development and physiological function-of mice and men. Genes Dev. 23, 788-797 (2009).

32. Rajakumari, S. et al. EBF2 determines and maintains brown adipocyte identity Cell Metab. 17, 562-574 (2013).

33. Puigserver, P. et al. A cold-inducible coactivator of nuclear receptors linked to adaptive thermogenesis. Cell 92, 829-839 (1998).

34. Kajimura, S. et al. Initiation of myoblast to brown fat switch by a PRD16-C/ EBP-beta transcriptional complex. Nature 460, 1154-1158 (2009).

35. Kajimura, S. et al. Regulation of the brown and white fat gene programs through a PRDM16/CtBP transcriptional complex. Genes Dev. 22, 1397-1409 (2008).

36. Pan, D., Fujimoto, M., Lopes, A. \& Wang, Y. X. Twist-1 is a PPARdeltainducible, negative-feedback regulator of PGC-1alpha in brown fat metabolism. Cell 137, 73-86 (2009).

37. Kiskinis, E. et al. RIP140 represses the "brown-in-white" adipocyte program including a futile cycle of triacylglycerol breakdown and synthesis. Mol. Endocrinol. 28, 344-356 (2014).

38. Zhang, Y. et al. Foxp1 coordinates cardiomyocyte proliferation through both cell-autonomous and nonautonomous mechanisms. Genes Dev. 24, 1746-1757 (2010)

39. Wang, B. et al. Foxp1 regulates cardiac outflow tract, endocardial cushion morphogenesis and myocyte proliferation and maturation. Development 131 , 4477-4487 (2004)

40. Li, S. et al. Foxp1/4 control epithelial cell fate during lung development and regeneration through regulation of anterior gradient 2. Development 139, 2500-2509 (2012).

41. Shu, W. et al. Foxp2 and Foxp1 cooperatively regulate lung and esophagus development. Development 134, 1991-2000 (2007).

42. $\mathrm{Hu}, \mathrm{H}$. et al. Foxpl is an essential transcriptional regulator of B cell development. Nat. Immunol. 7, 819-826 (2006).

43. Feng, X. et al. Transcription factor Foxp1 exerts essential cell-intrinsic regulation of the quiescence of naive T cells. Nat. Immunol. 12, 544-550 (2011).

44. Zou, Y. et al. Forkhead Box P1 (FOXP1) Transcription Factor Regulates Hepatic Glucose Homeostasis. J. Biol. Chem. 290, 30607-30615 (2015).

45. Zhao, H. et al. Foxp1/2/4 regulate endochondral ossification as a suppresser complex. Dev. Biol. 398, 242-254 (2015).

46. Usui, N. et al. Foxp1 regulation of neonatal vocalizations via cortical development. Genes Dev. 31, 2039-2055 (2017).

47. Frohlich, H., Rafiullah, R., Schmitt, N., Abele, S. \& Rappold, G. A. Foxp1 expression is essential for sex-specific murine neonatal ultrasonic vocalization. Hum. Mol. Genet 26, 1511-1521 (2017)

48. Rocca, D. L., Wilkinson, K. A. \& Henley, J. M. SUMOylation of FOXP1 regulates transcriptional repression via CtBP1 to drive dendritic morphogenesis. Sci. Rep. 7, 877 (2017).

49. Li, H. et al. FOXP1 controls mesenchymal stem cell commitment and senescence during skeletal aging. J. Clin. Invest 127, 1241-1253 (2017)

50. Wang, B., Lin, D., Li, C. \& Tucker, P. Multiple domains define the expression and regulatory properties of Foxp1 forkhead transcriptional repressors. J. Biol. Chem. 278, 24259-24268 (2003).

51. Zou, Y. et al. IRX3 Promotes the browning of white adipocytes and its rare variants are associated with human obesity risk. EBioMedicine 24, 64-75 (2017)

52. Wang, Q. et al. Brown adipose tissue in humans is activated by elevated plasma catecholamines levels and is inversely related to central obesity. PLoS ONE 6, e21006 (2011)

53. Gerin, I. et al. On the role of FOX transcription factors in adipocyte differentiation and insulin-stimulated glucose uptake. J. Biol. Chem. 284, 10755-10763 (2009)

54. Cao, W., Medvedev, A. V., Daniel, K. W. \& Collins, S. beta-Adrenergic activation of p38 MAP kinase in adipocytes: cAMP induction of the uncoupling protein 1 (UCP1) gene requires p38 MAP kinase. J. Biol. Chem. 276, 27077-27082 (2001).

55. Eguchi, J. et al. Transcriptional control of adipose lipid handling by IRF4. Cell Metab. 13, 249-259 (2011)

56. He, W. et al. Adipose-specific peroxisome proliferator-activated receptor gamma knockout causes insulin resistance in fat and liver but not in muscle. Proc. Natl Acad. Sci. USA 100, 15712-15717 (2003). 
57. Galmozzi, A. et al. ThermoMouse: an in vivo model to identify modulators of UCP1 expression in brown adipose tissue. Cell Rep. 9, 1584-1593 (2014).

58. Harms, M. J. et al. Prdm16 is required for the maintenance of brown adipocyte identity and function in adult mice. Cell Metab. 19, 593-604 (2014).

59. Collins, S., Yehuda-Shnaidman, E. \& Wang, H. Positive and negative control of Ucp1 gene transcription and the role of beta-adrenergic signaling networks. Int J. Obes. (Lond.) 34(Suppl 1), S28-S33 (2010).

60. Hansen, J. B. et al. Retinoblastoma protein functions as a molecular switch determining white versus brown adipocyte differentiation. Proc. Natl. Acad. Sci. USA 101, 4112-4117 (2004).

61. Sharma, B. K., Patil, M. \& Satyanarayana, A. Negative regulators of brown adipose tissue (BAT)-mediated thermogenesis. J. Cell Physiol. 229, 1901-1907 (2014).

62. Cohen, P. et al. Ablation of PRDM16 and beige adipose causes metabolic dysfunction and a subcutaneous to visceral fat switch. Cell 156, 304-316 (2014).

63. Krueger, K. C., Costa, M. J., Du, H. \& Feldman, B. J. Characterization of Cre recombinase activity for in vivo targeting of adipocyte precursor cells. Stem Cell Rep. 3, 1147-1158 (2014).

64. Cao, T. T., Deacon, H. W., Reczek, D., Bretscher, A. \& von Zastrow, M. A kinase-regulated PDZ-domain interaction controls endocytic sorting of the beta2-adrenergic receptor. Nature 401, 286-290 (1999).

65. Nantel, F. et al. The human beta 3-adrenergic receptor is resistant to short term agonist-promoted desensitization. Mol. Pharm. 43, 548-555 (1993).

66. Liggett, S. B., Freedman, N. J., Schwinn, D. A. \& Lefkowitz, R. J. Structural basis for receptor subtype-specific regulation revealed by a chimeric beta $3 /$ beta 2-adrenergic receptor. Proc. Natl. Acad. Sci. USA 90, 3665-3669 (1993).

67. Chen, Y. et al. Thermal stress induces glycolytic beige fat formation via a myogenic state. Nature 565, 180-185 (2019).

68. Bhide, A. A., Digesu, G. A., Fernando, R. \& Khullar, V. Use of mirabegron in treating overactive bladder. Int Urogynecol J. 23, 1345-1348 (2012).

69. Feng, X. et al. Foxp1 is an essential transcriptional regulator for the generation of quiescent naive T cells during thymocyte development. Blood 115, 510-518 (2009).

70. Zhang, X. L. et al. Integrative epigenomic analysis reveals unique epigenetic signatures involved in unipotency of mouse female germline stem cells. Genome Biol. 17, 162 (2016).

\section{Acknowledgements}

This work was supported by research grants from the National Natural Science Foundation of China [91749103, 81421061, 31100624 and 81200586] and grant from the National Major Fundamental Research 973 Program of China [2014CB942902] to X.Z.G., grants from the NIH (R01CA31534), Cancer Prevention Research Institute of
Texas (RP120348 and RP120459) and the Marie Betzner Morrow Centennial Endowment to H.O.T.

\section{Author Contributions}

P.L., S.H., S.L., S.X., F.W., W.Z. and R.Z. performed experiments, J.W. and X.G. designed experiments, C.H. and X.Z. helped ChIP-seq analysis, Y.F., N.W., L. H., X.X. and Z.Y. helped preparing samples and instructing experiments, H.T. and J.W. provided human biopsies and mouse lines and revised manuscript, X.G. wrote paper.

\section{Competing interests}

The authors declare no competing interests.

\section{Additional information}

Supplementary information is available for this paper at https://doi.org/10.1038/s41467019-12988-8.

Correspondence and requests for materials should be addressed to J.W. or X.G.

Reprints and permission information is available at http://www.nature.com/reprints

Peer Review Information Nature Communications thanks the anonymous reviewer(s) for their contribution to the peer review of this work. Peer reviewer reports are available.

Publisher's note Springer Nature remains neutral with regard to jurisdictional claims in published maps and institutional affiliations.

Open Access This article is licensed under a Creative Commons Attribution 4.0 International License, which permits use, sharing, adaptation, distribution and reproduction in any medium or format, as long as you give appropriate credit to the original author(s) and the source, provide a link to the Creative Commons license, and indicate if changes were made. The images or other third party material in this article are included in the article's Creative Commons license, unless indicated otherwise in a credit line to the material. If material is not included in the article's Creative Commons license and your intended use is not permitted by statutory regulation or exceeds the permitted use, you will need to obtain permission directly from the copyright holder. To view a copy of this license, visit http://creativecommons.org/ licenses/by/4.0/.

(C) The Author(s) 2019 